\title{
On the relationship between morphology and conductivity in nanosheet networks
}

\author{
Sebastian Barwich a, b, João Medeiros de Araújo ${ }^{c}$, Aran Rafferty ${ }^{\text {a }}$, \\ Claudia Gomes da Rocha ${ }^{\mathrm{d}}$, Mauro S. Ferreira ${ }^{\mathrm{a}, \mathrm{b}}$, Jonathan N. Coleman ${ }^{\mathrm{a}, \mathrm{b}, \text { * }}$ \\ ${ }^{\text {a } C e n t r e ~ f o r ~ R e s e a r c h ~ o n ~ A d a p t i v e ~ N a n o s t r u c t u r e s ~ a n d ~ N a n o d e v i c e s ~(C R A N N) ~ \& ~ A d v a n c e d ~ M a t e r i a l s ~ a n d ~ B i o e n g i n e e r i n g ~ R e s e a r c h ~(A M B E R) ~ C e n t r e, ~ T r i n i t y ~}$ \\ College Dublin, Dublin 2, Ireland \\ ${ }^{\mathrm{b}}$ School of Physics, Trinity College Dublin, Dublin 2, Ireland \\ ${ }^{c}$ Departamento de Física Teórica e Experimental, Universidade Federal Do Rio Grande Do Norte, Natal, RN, 59078-900, Brazil \\ d Department of Physics and Astronomy, University of Calgary, 2500 University Drive NW Calgary, Alberta, T2N 1N4, Canada
}

\section{A R T I C L E I N F O}

\section{Article history:}

Received 2 July 2020

Received in revised form

28 August 2020

Accepted 3 September 2020

Available online 7 September 2020

\section{Keywords:}

Nanosheet

Network

Morphology

Conductivity

\begin{abstract}
A B S T R A C T
It is well-known that the morphology of nanostructured networks is closely linked to network properties. However, controlling and characterizing the morphology of networks of 2D nanosheets has not been explored. In this work, we use networks of liquid-exfoliated graphene nanosheets as a model system to examine the relationship between network morphology and conductivity in nanosheet networks. We use a combination of heat and pressure to controllably alter the morphology of the network, resulting in the annihilation of large pores $(>40 \mathrm{~nm}$ ) and improved nanosheet alignment within the sample. Such compression can result in a tenfold increase in network conductivity. Analysis shows both in-plane and out-of-plane conductivities to scale with porosity in line with percolation theory. The conductivity anisotropy was 3000 at low-porosity and was projected to fall to 1 in the limit of high porosity. Computational studies link the conductivity increase to an increase in network connectivity and a reduction in junction resistance as the porosity is decreased.
\end{abstract}

(C) 2020 Elsevier Ltd. All rights reserved.

\section{Introduction}

In recent years, liquid phase exfoliation (LPE) has become a relatively common method for producing nanosheets of both graphene and a range of other 2D materials [1-3]. This method involves the sonicating or shearing of layered crystals in solvents or aqueous surfactant solutions resulting in the formation of dispersions of stabilized nanosheets. It is a flexible [4-7] and scalable [8] method that has been used to produce a large variety of 2D materials including graphene, $\mathrm{BN}, \mathrm{MoS}_{2}, \mathrm{GaS}$ and phosphorene [3,9-13]. The main disadvantage of this technique is that the resultant nanosheets tend to be polydisperse and can have relatively low aspect ratio [14].

One of the main practical applications of liquid-dispersed nanosheets is the ease with which they can be formed into

\footnotetext{
* Corresponding author. Professor of Chemical Physics, School of Physics, CRANN Institute and AMBER, the SFI Research Centre for Advanced Materials and BioEngineering Research, Trinity College Dublin, Ireland.

E-mail address: colemaj@tcd.ie (J.N. Coleman).
}

composites [15] or nanosheet networks [16] which can then be used for a number of applications from sensors to electronic devices [16-27]. Such networks can be formed in a variety of ways, from drop-casting [28], to filtration [13,29], or printing $[16,26,27,30-33]$. The range of such applications is quite broad, simply because of the diversity of the 2D materials which can be produced by liquid exfoliation. For example, due to its electronic conductivity, networks of liquid-exfoliated graphene nanosheets can be used as printed electrodes [16,27,34,35]. Conversely, due to their insulating nature BN networks can be used as ion conductors $[16,36]$ or dielectrics [37]. Alternatively, the semiconducting nature of materials such as $\mathrm{MoS}_{2}$ or $\mathrm{WSe}_{2}$ has allowed their networks to be used as the active material in transistors [16,34,38]. Finally, their electrochemical properties has allowed networks of 2D materials such as $\mathrm{GeS}$ and $\mathrm{Ni}(\mathrm{OH})_{2}$ to be used in applications such as battery electrodes [39] or electrocatalysts [40].

In general, nanosheet networks consist of disordered arrays of nanosheets which often have a partial degree of in-plane alignment [26]. Such networks are always quite disordered and display a wide range [41] of morphologies from highly porous [16] to closely 
packed $[38,42]$. The morphology tends to vary with deposition method, the specific conditions during deposition and the properties of the nanosheets being deposited. For example, in printed films, morphology can vary significantly with ink formulation [41], particle size [43], spray droplet size [44,45] and drying conditions [46-48].

The network morphology is particularly important because it determines the nature of the junctions connecting nanosheets. These junctions represent the locations where adjacent nanosheets are in close proximity, separated only by a closely spaced van der Waals (vdW) gap. Although their properties have not been closely studied, one can imagine a number of junction types, for example: plane-plane, plane-edge, plane-vertex, edge-edge etc. Charge transport across these junctions is expected to be via quantum tunneling or hopping across the vdW gap [16]. In the case of networks of graphene nanosheets the conductivity is limited by charges moving across junctions via hopping processes with temperature-dependent measurements showing evidence of variable range hopping [49] or Arrhenius-like behavior [31].

We expect the exact details of the junctions to determine the effective inter-nanosheet junction resistance which in turn limits the network mobility. Previous work has shown the mobility of networks of thick, rigid $\mathrm{MoS}_{2}$ nanosheets to be relatively low $\left(\sim 10^{-3}-10^{-2} \mathrm{~cm}^{2} / \mathrm{Vs}\right)[16,34,50,51]$. This is probably because rigid platelets become jammed during network formation [52], resulting in low-area, point-like contacts. Temperature dependent measurements in such samples show Arrhenius-like behavior, consistent with inter-nanosheet hopping, with large $(\sim 0.2-0.3 \mathrm{eV})$ activation energies [16]. Alternatively, it has been shown that very thin, flexible $\mathrm{MoS}_{2}$ nanosheets, can form intimately connected, large area junctions resulting in high network mobilities $\left(>1 \mathrm{~cm}^{2}\right.$ ) Vs) [38]. Such high mobilities must be due to the low junction resistance associated with the well-connected, large-area contacts. We speculate that these systems are still limited by hopping between nanosheets but that the closely spaced nanosheets yield a much-reduced activation energy.

Similarly, morphology is known to play a role in the conductivity of graphene nanosheet networks [41]. In the case of films of highaspect ratio graphene sheets, annealing and densification from 1.2 to $1.8 \mathrm{~g} / \mathrm{cm}^{3}$ resulted in a large conductivity increase from $1.7 \times 10^{4} \mathrm{~S} / \mathrm{m}$ to $2.2 \times 10^{5} \mathrm{~S} / \mathrm{m}$, probably as a result of increased junction overlap area [53]. However, beyond these basic facts, very little is known about nanosheet network morphology, meaning we have very limited understanding of the factors linking network structure to conductivity.

In this work we use liquid-exfoliated graphene nanosheets as a model system to explore the effects of nanosheet network morphology on electrical conductivity. To do this, we produce porous nanosheet networks which we then controllably compress, changing the porosity, leading to variations in network morphology and ultimately conductivity. We then use a combination of experimental characterization and theoretical modeling to shed light on the relationship between conductivity and morphology in nanosheet networks.

\section{Experimental section}

Experimental methods: Graphite was sourced from Asbury Graphite grade 3763. N-methyl-pyrolidone (NMP) was purchased from Sigma Aldrich (>99\% purity) and used as received. Shear exfoliation [8] of graphite in NMP at $50 \mathrm{~g} / \mathrm{L}$ was performed at $6000 \mathrm{rpm}$ for $6 \mathrm{~h}$ using a Silverson high-shear mixer (model L5M-A, $746 \mathrm{~W}$ ) using the as-supplied standard large rotor-stator mixing assembly. Dispersions were centrifuged $1000 \mathrm{rpm}$ for $90 \mathrm{~min}$ using a Haraeus Megafuge 16 using a TX-400 swinging bucket rotor (max
$5000 \mathrm{rpm})$. Graphene suspensions were obtained from the supernatant by decantation while the sediment was recycled as a starting material for further exfoliation batches.

TEM samples were prepared via drop casting of the liquid exfoliated sample onto holey carbon grids and the micrographs were obtained using a JEOL 2100. For SEM and Raman samples were prepared by vacuum filtration through a porous nylon membrane (pore size $0.1 \mu \mathrm{m}$ ). The films were dried overnight under vacuum at $70{ }^{\circ} \mathrm{C}$ and were free standing from the membrane. Raman was performed using a Horiba Jobin LabRAM equipped with a $100 \times$ objective lens and a $633 \mathrm{~nm}$ laser excitation. An average of 10 spots were measured across the sample surface, the baseline was removed and the spectra averaged.

In order to form the nanosheet networks, the graphene dispersion was filtered through a nano-porous nylon membrane (Whatman $0.2-\mu \mathrm{m}$ pore size) and the film removed from the membrane and dried overnight in a vacuum oven at $60^{\circ} \mathrm{C}$. Strips of film of $\sim 18 \mathrm{~cm}^{2}$ area were then compressed in a hydraulic press at various pressures between $\sim 6$ and $\sim 40 \mathrm{MPa}$ and held under compression for $10 \mathrm{~min}$ at $150{ }^{\circ} \mathrm{C}$. We note that preliminary measurements showed the resistance obtained after pressing at $150{ }^{\circ} \mathrm{C}$ to be roughly $30 \%$ lower than that achieved at room temperature, a factor which we attribute to the removal of residual solvent (either NMP or adsorbed water). Film dimensions and weight were measured before and after compression.

Porosity was calculated with respect to the density of solid graphite $\left(2200 \mathrm{~kg} / \mathrm{m}^{3}\right)$. SEM micrographs were obtained using a Zeiss Ultra with an accelerating voltage of $5 \mathrm{kV}$. Orientation distributions were obtained using the "OrientationJ" plugin for "ImageJ" image analysis software. The SEM micrographs analysed were all of equal magnification ( $\times 3 \mathrm{k}$ ) except for three thicker uncompressed samples which had a slightly lower magnification. Images were contrast-corrected and cropped to only include infocus areas of the fracture surface prior to extracting orientation distributions. Multiple SEM micrographs were analysed, fitted using a structural tensor with a 20-pixel local window and a Gaussian gradient. The resulting distribution was fitted to a Gaussian peak and the FWHM extracted and averaged. Mercury porosimetry was performed using an Autoscan-33 Porosimeter (Quantachrome, UK). BET analysis performed using a Nova 2400e surface area and pore size analyser (Quantachrome, UK). Conductivity measurements were performed by cutting strips from the compressed and uncompressed films, painting on silver contacts and measuring current/voltage sweeps using a Keithly 2400 . A minimum quantity of silver paint was used to minimize the effects of silver infiltration into the pores but yield a reliable contact. However, we note that, in porous samples it is very hard to completely eliminate such infiltration. However, the thickness of the samples (always $>100 \mu \mathrm{m}$ ) was such that the effects of silver infiltration should be minimal. In addition, the most porous samples are also the thickest samples (thickness of $\sim 300 \mu \mathrm{m}$ ). In such highly porous sample, while the absolute degree of infiltration would be greatest, the fractional distance over which silver can infiltrate may be comparable or even lower than less porous samples.

Deposition simulation: The deposition simulations were run with the Open Dynamic Engine (ODE) library [SMITH, R., 2006. Open dynamics engine (ODE); http://www.ode.org], which describes the motion of classical objects under the relevant force fields. It enables us to simulate rigid body dynamics of 3D objects using advanced joint types and integrated collision detection including friction. During the simulation, collisions between moving bodies or between bodies and the static environment are performed and calculated for a series of time steps. Full information about the locations of all objects is fully available throughout the simulation. Deposition is complete when all platelets are in their resting 
positions which are uniquely defined by positioning their individual centres of mass together with the coordinates of a vector that is normal to the squared face of each platelet. From these data a series of subsequent calculations can be carried out.

Porosity calculation: a box of volume Vbox $=100 \times 100 \times 1.6$ units $^{3}$ used as a reference for all calculations. The computational units are arbitrary. From the position of each platelet it is straightforward to obtain the porosity by simply calculating the volume fraction taken by the platelets that fall inside the reference box.

Platelet orientation: The orientation is obtained from the angle formed by the normal vector of the platelet with respective to the transverse direction of the box. A distribution of such angles describes the degree of alignment between platelets.

Platelet Contacts: To identify if two platelets are in contact, it is sufficient to test whether any one of the six vertices of a platelet lies inside a cuboid defined by the six vertices of another block. In other words, the objects are allowed a minute amount of interpenetration, which is controllable in the ODE library. To avoid extensive calculations, we only perform this verification if two blocks are close enough.

Contact area: Contact area between two platelets is found by projecting the interpenetration volume on the faces of the platelets. First, we identify if two platelets are in contact (see above) and then project the corresponding interpenetrated volume on the larger face of either platelet. The maximum contact area, in this way, will be the area of the larger face.

Conductivity calculation: The network formed by junction resistances is transformed into a mathematical graph [54,55] that is subsequently used to write Kirchhoff's laws in terms of the Laplacian matrix describing the connectivity of the network. Minimum residual iteration is used to solve a system of linear circuit equations to obtain the conductance betweenany two voltage nodal points of the network, which also enables us to find the corresponding conductivity.

\section{Results and discussion}

\subsection{Basic characterization}

The graphene nanosheets used in this work were produced by shear-assisted liquid phase exfoliation (LPE), a commonly used technique for producing nanosheets in large quantities [8]. A TEM image of a typical nanosheet is shown in Fig. $1 \mathrm{~A}$ with a flake length histogram shown in Fig. 1B. The distribution is relatively broad with nanosheet of up to $1200 \mathrm{~nm}$ long observed and a mean nanosheet length of $260 \mathrm{~nm}$. We note that shear exfoliation under conditions such as these tends to yield graphene nanosheets with mean
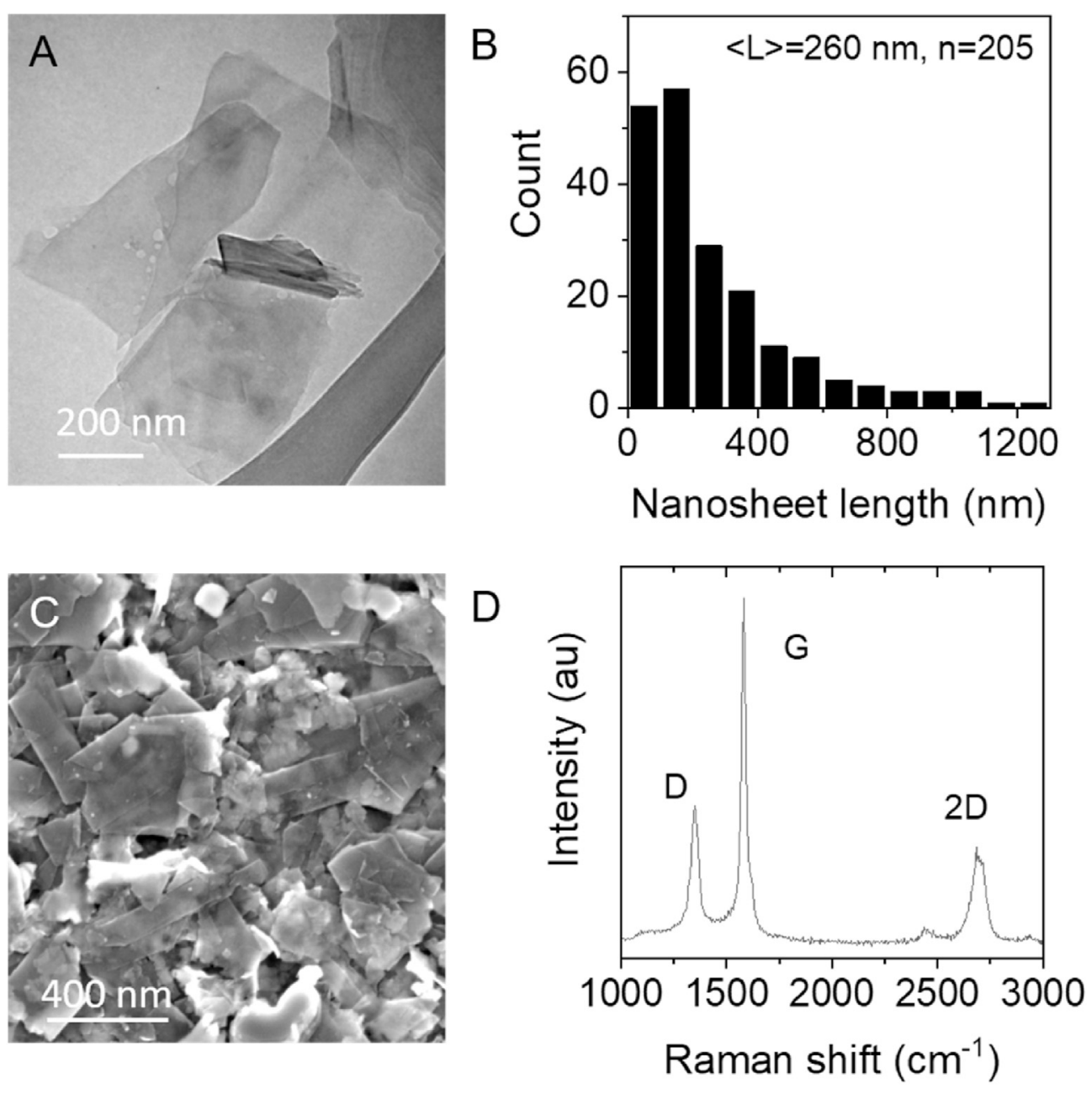

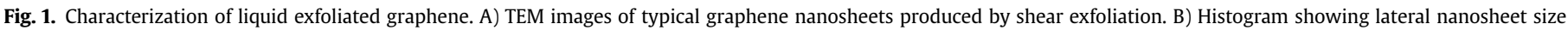

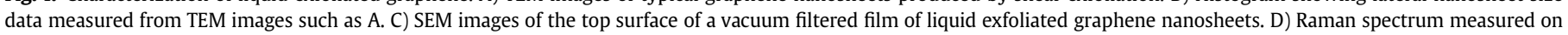
the surface of a filtered graphene film with the D, G and 2D modes indicated. 
thickness of $\sim 6-9$ monolayers [8]. Liquid exfoliated nanosheets can easily be formed into films by vacuum filtration (Fig. 1C). Such films consist of highly disordered networks of nanosheets which are somewhat aligned in the plane of the film. A Raman spectrum of a film of graphene nanosheets is shown in Fig. 1D. This spectrum is typical of liquid exfoliated graphene and displays the fingerprint $\mathrm{D}$, $\mathrm{G}$ and $2 \mathrm{D}$ bands. We note that it has been shown in a number of papers $[8,56]$ that the relatively small D-band displayed by LPE graphene is not evidence of basal plane defects but rather is associated with edge defects.

Here we are interested in the properties of nanosheet networks such as that in Fig. 1C. Although it is possible to controllably vary network porosity during film formation for nanosheet networks [41], here we decided to use a post-treatment technique to modify the porosity of existing films via pressure and heat. To achieve this, we first prepared relatively thick $(\sim 300 \mu \mathrm{m})$ vacuum-filtered films of liquid exfoliated [57] graphene nanosheets (see methods) which naturally contain a porosity of $\sim 70 \%$. These films were then hotpressed at a variety of pressures, reducing the film thickness by an amount, $\Delta t$, relative to the initial thickness, $t_{0}$. The most highly compressed films were $\sim 100 \mu \mathrm{m}$ thick. The resultant compression ratio, expressed as $-\Delta t / t_{0}$, is shown as a function of pressure in Fig. $2 \mathrm{~A}$ and reached $\sim 70 \%$ for $65 \mathrm{kN}$. Measurements of film mass and dimensions after pressing yielded the density, $\rho_{\mathrm{Net}}$, which can be used to estimate the film porosity, $\mathrm{P}$, via $P=1-\rho_{N e t} / \rho_{N S}$, where $\rho_{\mathrm{NS}}=2200 \mathrm{~kg} / \mathrm{m}^{3}$ is the nanosheet density. As shown in Fig. $2 \mathrm{~B}$, this yielded films with porosity in the range $70 \%-14 \%$.

We note that pressure treatments have been performed before [26,27], for example on films of graphene oxide nanosheets [58]. That work used high pressures of up to $300 \mathrm{MPa}$ to achieve pressure-assisted thermal graphitization of the nanosheets thus converting graphene oxide to a much more graphene-like material. These structural changes resulted in a significant conductivity enhancement. However, in that case, the major effect of pressure and temperature was probably the chemical alteration of the individual nanosheets with structural arrangements of the network a secondary effect. Here, we used lower pressures of $<50 \mathrm{MPa}$. In addition, the LPE graphene nanosheets are already free of basal plane defect and oxides (see Raman spectrum in Fig. 1D) and should display the full mechanical properties of graphene. Thus, we believe the nanosheets themselves are unlikely to be damaged significantly by the pressing procedure. However, it is likely that the network morphology will be significantly changed, and this will dominate any change to conductivity.

In order to investigate the effects of compression, SEM was performed on fracture surfaces with examples of high and low
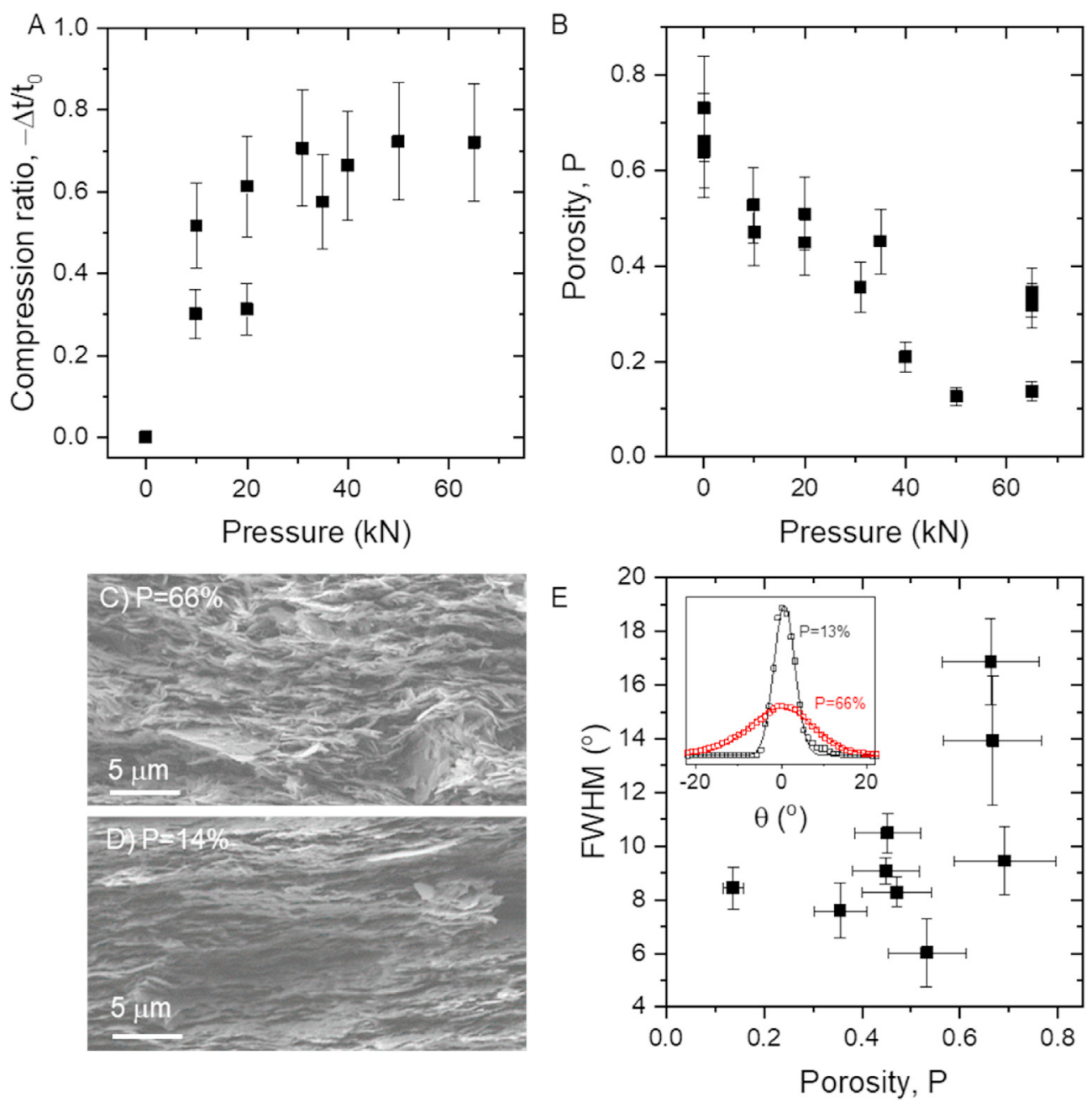

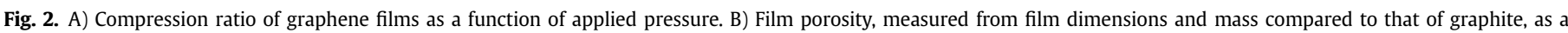

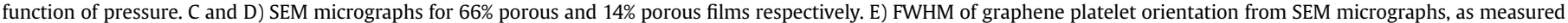

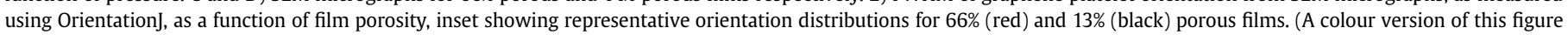
can be viewed online.) 
porosity films shown in Fig. 2C and D. Here, the effects of compression can be seen with the uncompressed samples showing a greater range of platelet orientation whereas the compressed samples appear to exhibit more in-plane alignment. To characterize the effect of pressing on nanosheet orientation more quantitatively, image analysis was performed using OrientationJ, a plugin for ImageJ image analysis software. Here the apparent orientation of the platelet was measured relative to the horizontal. This allowed

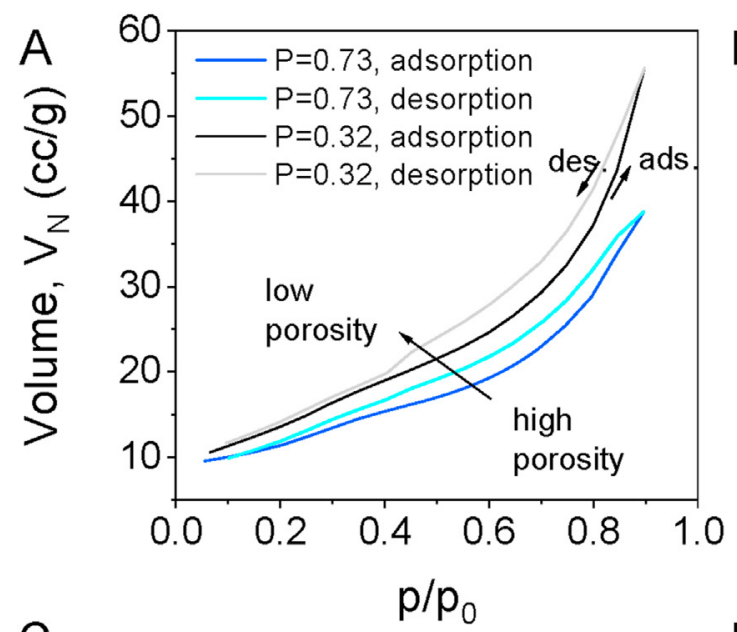

$\mathrm{B}$

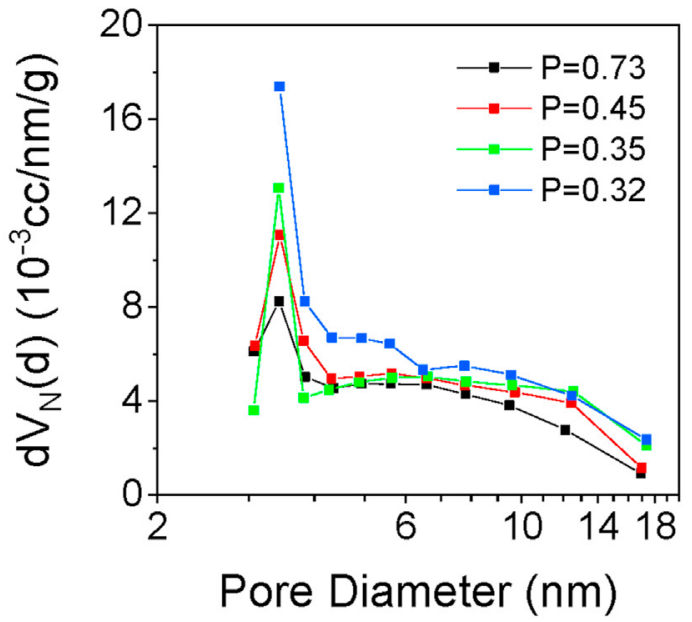

C
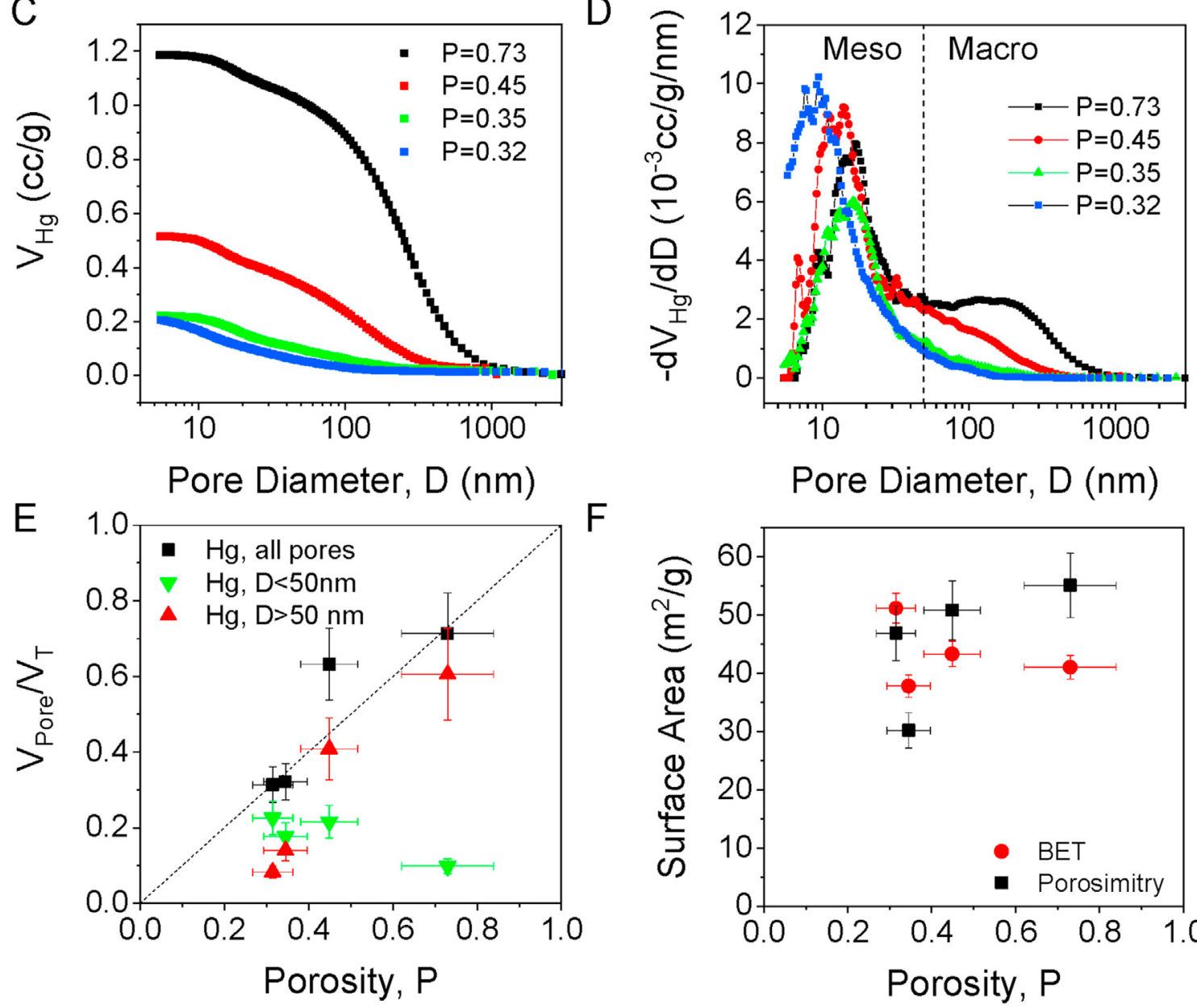

$\mathrm{F}$

Pore Diameter, D $(\mathrm{nm})$

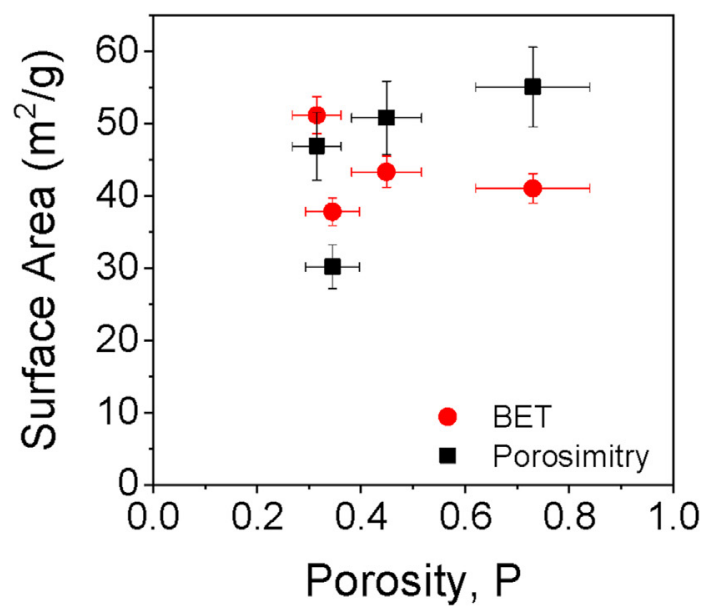

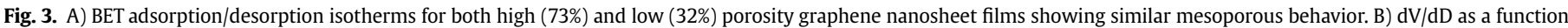

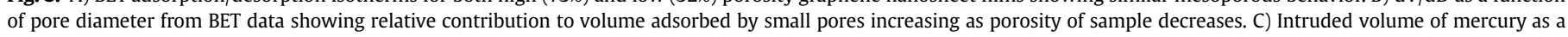

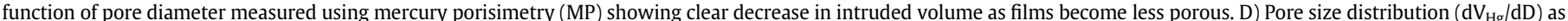

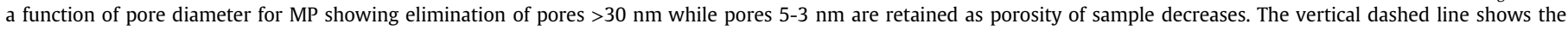

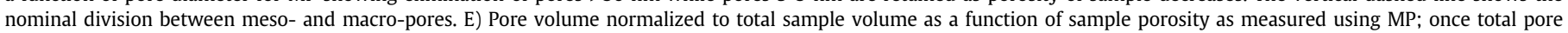

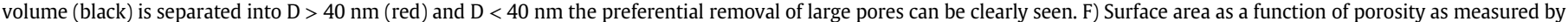

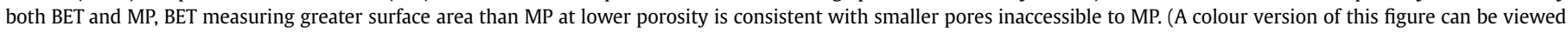
online.) 
the orientation distribution of the platelets to be estimated as illustrated in the inset of Fig. 2E. However, we note that the waviness of the nanosheets may result in an over-estimation of the orientation distribution as measured in this way. In all cases the distributions were Gaussian in shape (solid lines) with the widths (FWHM) plotted versus porosity in Fig. 2E. Although the data is scattered it is clear that the distribution width increases with increasing porosity. This is consistent with a pressure-induced transformation from a relatively open structure to a more closed structure characterized by a greater degree of nanosheet orientation in the plane of the film.

\subsection{Porosimetry}

In addition to the porosity, it would be of interest to know more about the distribution of pore sizes in the network and how this distribution is affected by compression. To investigate this, we turn to the nitrogen adsorption/desorption isotherms (i.e. BET analysis) and mercury intrusion porosimetry (MP). Both BET and MP measurements were made on 4 graphene nanosheet films with porosities, $\mathrm{P}$, of $0.32,0.35,0.45$ and 0.73 . Shown in Fig. $3 \mathrm{~A}$ are examples of nitrogen adsorption and desorption isotherms for the highest and lowest porosity films. In all cases, the isotherms were of type IV, characterized by considerable hysteresis, consistent with the occurrence of nitrogen condensation in mesopores (i.e. those pores with size in the range $2-50 \mathrm{~nm}$ ) [59]. In addition, the shape of the hysteresis loop is consistent with the type H3 loop. Such loops are known to occur for materials consisting of aggregates of platelets which yield slit-like pores, as would be expected for nanosheet networks [59]. Shown in Fig. 3B are pore-size distributions estimated from the BET curves. These clearly show the presence of mesopores but do not show any significant difference between the different porosity films.

However, the mesopores observed by nitrogen absorption (Fig. 2B) could not represent the entire pore volume within these nanosheet networks. Integrating the curves in Fig. 3B gives a pore volume of $\sim 0.07 \mathrm{cc} / \mathrm{g}$ which is equivalent to porosity of $\sim 13 \%$, well below the measured value for most of the films under study.

In fact, it is likely that much larger pores are present which are not observable using nitrogen adsorption measurements. To address this, we use mercury intrusion porosimetry which can characterize pores up to $400 \mu \mathrm{m}$ in size [60]. Shown in Fig. 2C are graphs of intruded mercury volume as a function of pore size for graphene nanosheet films with four different porosities and clearly shows a significant trend as the porosity is decreased. The data in Fig. $3 \mathrm{C}$ can be converted to give a pore size distribution by differentiation $[59,61]$ as shown in Fig. 3D. This graph clearly shows all films to contain significant volumes of mesopores, irrespective of porosity. However, it also shows the $\mathrm{P}=0.73$ film to have a large volume of macropores (pores with size above $50 \mathrm{~nm}$ ). The contribution of macropores appears to fall with decreasing porosity with the $P=0.32$ film having a relatively small fraction of macropores.

This can be seen more clearly by quantitative analysis of the data in Fig. 3 C-D. Integrating the pore size distributions (Fig. 3D) over pore diameter yields the total pore volume in $\mathrm{cc} / \mathrm{g}$ (equivalent to the low-D plateaus in Fig. 2C). This is a measure of the pore volume divided by the sample mass $\left(\mathrm{V}_{\mathrm{P}} / \mathrm{M}\right)$ which can be related to the total film volume, $V_{\mathrm{T}}$, using: $V_{P} / M=\left(V_{P} / V_{T}\right) / \rho_{\text {Net }}$. This allows us to calculate the fractional pore volume $V_{P} / V_{T}$ (which should be equivalent to the porosity) using the measured film densities. Shown in Fig. 3E is data (black squares) for $V_{P} / V_{T}$ plotted versus $\mathrm{P}$ (estimated from measured film mass and volume). This graph shows good agreement supporting the accuracy of the MP data. Indeed, the agreement between porosity-values from porosimeter and density measurements would imply the pore structure to be

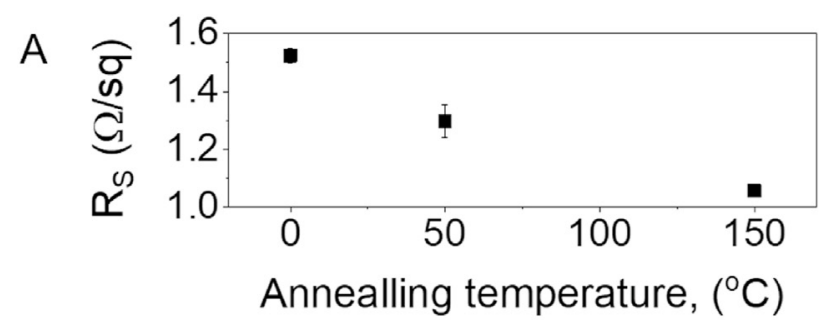

B
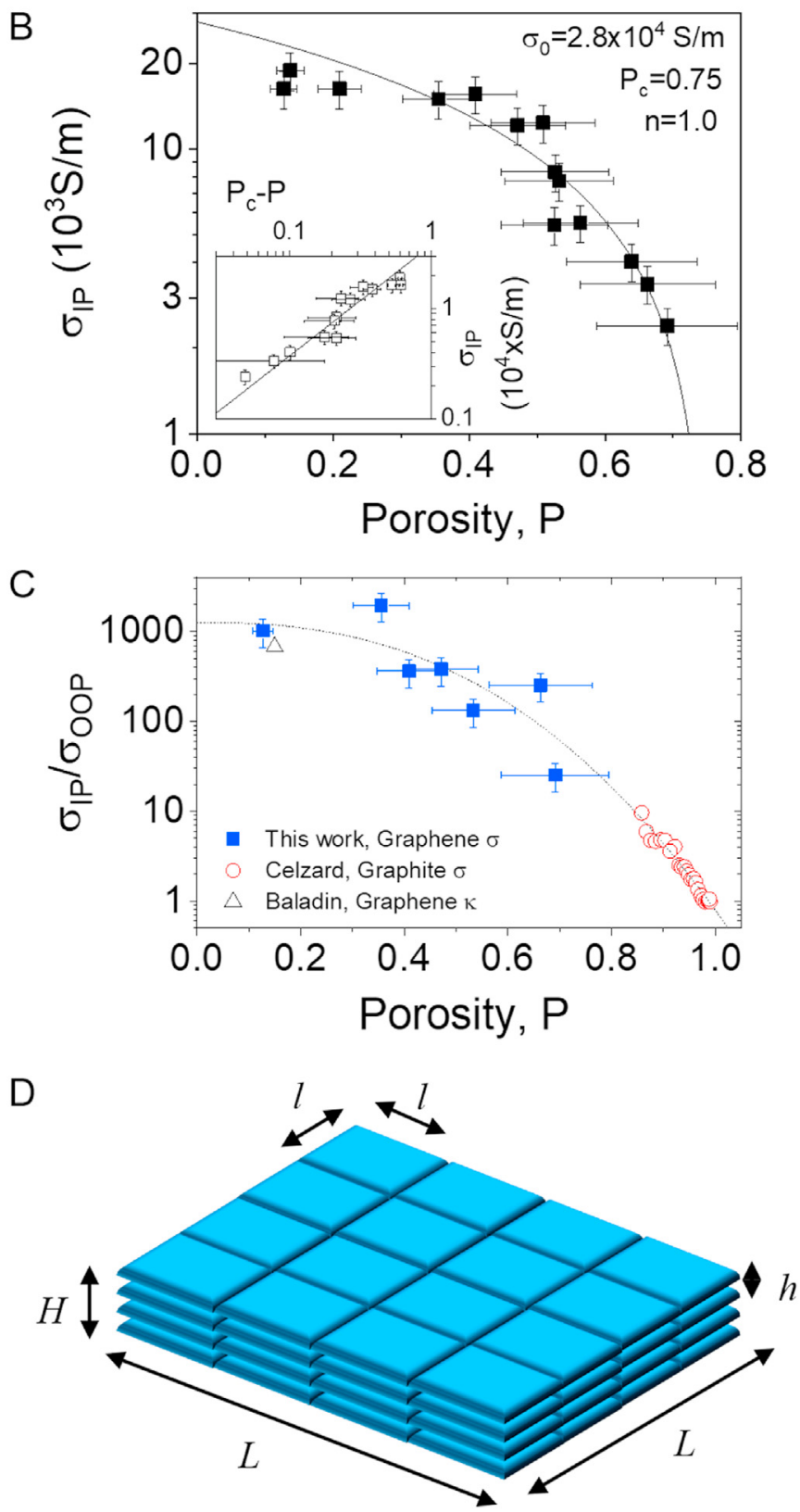

Fig. 4. A) Sheet resistance of graphene nanosheet films annealed at different temperatures. B) In-Plane film conductivity, $\sigma_{\mathrm{IP}}$, as a function of film porosity, P. Inset: Percolation plot of $\sigma_{\mathrm{IP}}$ Vs. P $\mathrm{P}_{\mathrm{C}}-\mathrm{P}$ fitted by percolation theory. The percolation fit has been reproduced in the main figure. C) In-plane/Out-of-plane conductivity ratio as a function of film porosity (blue squares). The data can be seen to be in agreement with that of Celzard et al. [62] for expanded graphite at different degrees of compression (open circles) as well as Balandin's data [72] for thermal conductivity anisotropy in graphene networks. The dashed line is an empirical curve which matches both data sets (see text). D) Schematic showing idealized layout of a zero-porosity network. (A colour version of this figure can be viewed online.) 
predominately open with very little contribution from closed pores.

Using a similar procedure we can estimate the contributions to $V_{P} / V_{T}$ from both meso- and macro-pores (for the former by integrating the distribution curve in the range below $\mathrm{D}=50 \mathrm{~nm}$ and for the latter by integrating in the range above $\mathrm{D}=50 \mathrm{~nm}$ ). These data are also plotted versus $\mathrm{P}$ in Fig. 3E. This clearly shows the contribution of the mesopores to be roughly constant at $20 \%$. However, it clearly shows the contribution of macropores to fall significantly with decreasing porosity. This data clearly shows that reducing the overall porosity by compression involves the annihilation of macropores while mesopores are hardly affected.

This conclusion is supported by data in Fig. 3E which shows the calculated sample surface area (both from BET and MP) as a function of porosity. The surface area falls only slightly as the porosity is decreased. This is as expected because the annihilation of the pore volume associated with macropores should not result in a large loss in surface area as small pores should have a much greater contribution of surface area per pore volume.

\subsection{Measurement of electrical properties}

Before assessing the effect of compression on the electrical properties of the films, it is worth briefly touching on the effect of temperature. To test this, we measured the sheet resistance of graphene films pressed at fixed pressure but at annealing temperatures of 0,50 and $150{ }^{\circ} \mathrm{C}$. We found a non-trivial decrease in sheet resistance with increased pressing temperature (Fig. 4A), with sheet resistance falling from $\sim 1.5 \Omega /$ sq to $\sim 1 \Omega /$ sq over the accessible temperature range. We believe that this resistance reduction is associated with the displacement of residual solvent (either NMP or water) during annealing, leading to the reduction of the inter-nanosheet junction resistance. All subsequent measurements were made at a temperature of $150{ }^{\circ} \mathrm{C}$.

It is clear from the data in Fig. 3 that the reduction of porosity achieved via compressing the films leads to significant morphological changes. We would expect such structural differences to result in a significant dependence of electrical conductivity on porosity. Shown in Fig. 4B are data for the electrical conductivity, measured in the plane of the films $\left(\sigma_{\mathrm{IP}}\right)$, plotted as a function of porosity. As the porosity was decreased, the conductivity increased from $2.3 \mathrm{kS} / \mathrm{m}$ for $\mathrm{P}=70 \%$ to $18.5 \mathrm{kS} / \mathrm{m}$ for the $\mathrm{P}=14 \%$ sample. This increase in conductivity with increasing pressure during compression is important as it indicates that the predominate changes in the film on compression are those associated with network morphology. If, for example, compression resulted in the fracture of the graphene nanosheets themselves, we would expect extra inter-nanosheet junctions to be introduced into the current paths which would likely reduce the conductivity.

It has previously been suggested that the conductivity of porous graphitic samples can be analysed using percolation theory [62]. This theory is usually used to analyze the conductivity of conductor-insulator composites as a function of conductor volume fraction, $\varphi$. In porous samples, the pores play the role of the insulator allowing the volume fraction of conductor to be found from $\varphi=1-\mathrm{P}$. In percolation theory, the overall conductivity is written as [63].

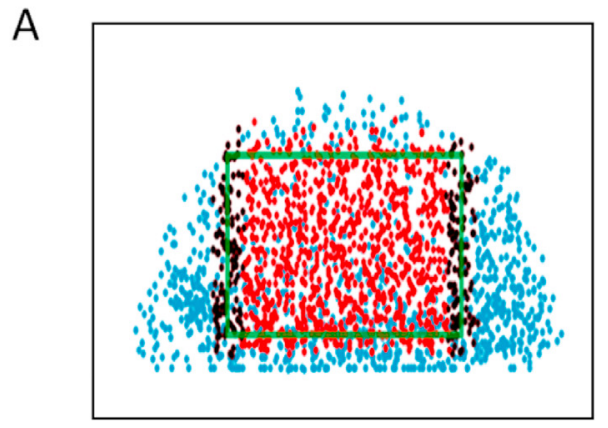

B
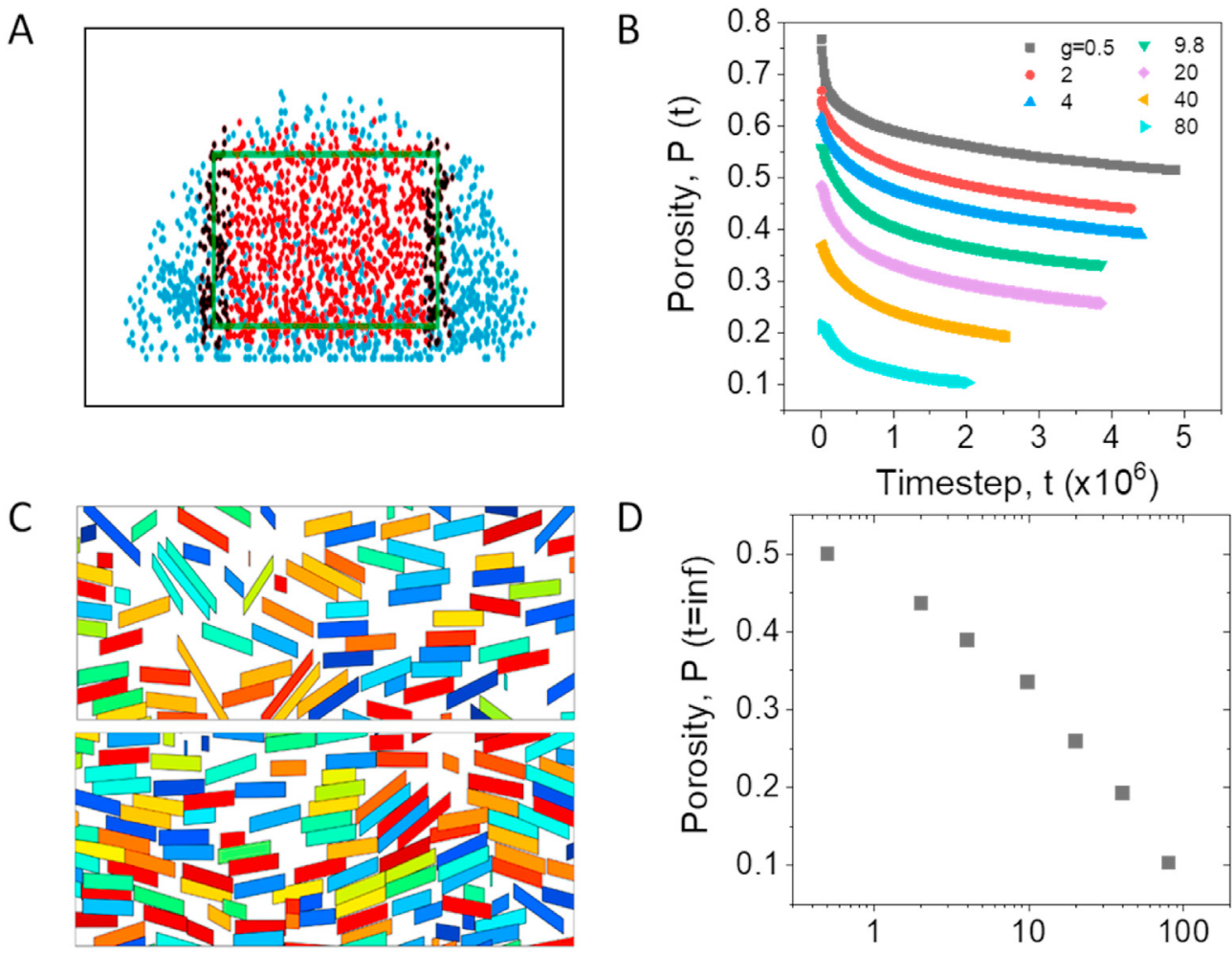

D

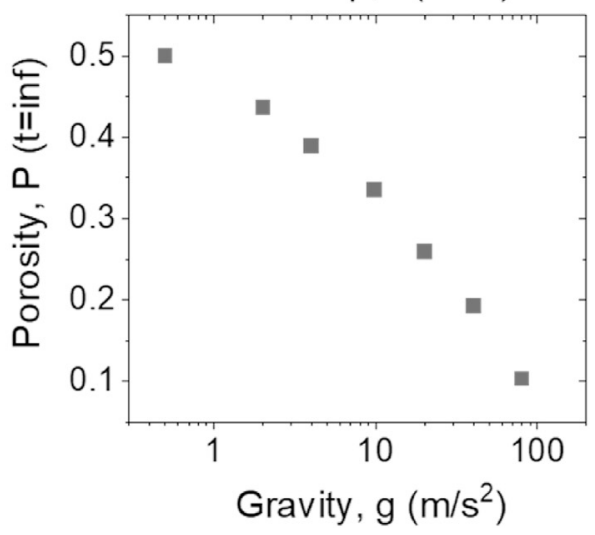

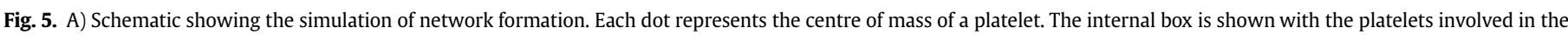

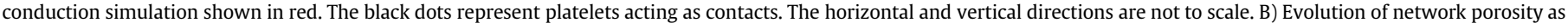

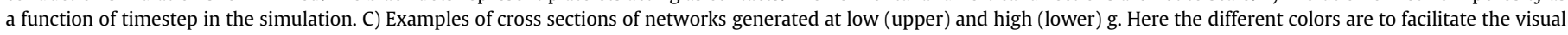

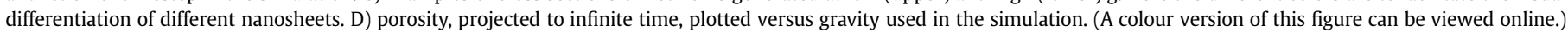


$\sigma=\sigma_{0}\left(\frac{\varphi-\varphi_{c}}{1-\varphi_{c}}\right)^{n}$

where $\sigma_{0}$ is the conductivity of a zero-porosity network, $\varphi_{c}$ is the minimum volume fraction where the network remains electrically connected (often termed the percolation threshold) and $n$ is the percolation exponent. When $\varphi_{c}$ is small, the denominator within the brackets is often neglected. However, this may not be the case here.

We can rewrite Eq. (1a) in terms of porosity using $\varphi=1-P$, obtaining:

$\sigma=\sigma_{0}\left(\frac{P_{c}-P}{P_{c}}\right)^{n}$

where $\mathrm{P}_{\mathrm{c}}$ is the critical porosity, that is the maximum porosity where a conducting path throughout the network exists. As shown in Fig. $4 \mathrm{~B}$, Eq. (1b) provides a reasonable fit to the data, yielding $\sigma_{0}=2.8 \times 10^{4} \mathrm{~S} / \mathrm{m}, \mathrm{P}_{\mathrm{c}}=0.75$ and $\mathrm{n}=1.0$. The zero-porosity conductivity $\left(\sigma_{0}\right)$ is well below the in-plane conductivity of graphite $\left(>10^{6} \mathrm{~S} / \mathrm{m}\right)$ [62] indicating that the network conductivity is limited by inter-nanosheet junctions. In addition, the critical porosity $\left(\mathrm{P}_{\mathrm{C}}\right)$ is quite low. In comparison, aero-graphite has shown non-zero conductivity at much higher porosities $\left(1-\mathrm{P} \sim 10^{-4}\right)$ [64]. However, we note that such networks show randomly oriented sheets which allow electrical connectivity even at very high porosities. In contrast, partially aligned networks such as those here are known to have relatively high percolation thresholds [63] and so relatively low critical porosities. The percolation exponent is reasonably close to the universal value of 1.3 expected for 2-dimensional percolation (i.e. in a thin film) consistent with the sample geometry (NB the universal percolation exponent for transport in three dimensions is 2.0) [65]. We note that percolation exponents tend to approach their universal values for nanoparticle networks where the particles are in close contact such that inter-particle junctions are vdW gaps [15]. In contrast, polymer-based composites tend to have junctions containing thin polymer layers of varying thickness [66]. Such polymer layers result in a wide range of junction resistances which manifests itself as a percolation exponent which is considerably higher than the universal one [66-68]. Thus, the low percolation exponent observed here implies that the distribution of junction resistances is quite narrow in these samples.

It is possible to estimate a lower limit for the mean junction resistance for these networks. The low-porosity network conductivity is $2 \times 10^{4} \mathrm{~S} / \mathrm{m}$, a factor of $\sim 100$ lower than the in-plane conductivity of graphite, a difference which we associated with inter-nanosheet junctions. If we assume that: firstly, in the limit of zero junction resistance, the in-plane network conductivity would approach that of graphite; and secondly, that the junction resistance $\left(R_{J}\right)$ is much larger than the nanosheet resistance, it should be approximately true that: $\sigma_{\text {graphite }} / \sigma_{\text {network }} \sim R_{\mathrm{J}} / R_{\text {nanosheet }}$. Modeling the nanosheet as square with thickness, t, yields: $R_{\text {nanosheet }} \sim 1$ / $\sigma_{\text {graphite }} \times t$. Combining and rearranging gives: $R_{J} \sim 1 / \sigma_{\text {network }} \times t$. Taking a typical nanosheet thickness of a few $n m$ yields an estimate of $R_{J} \sim 10 \mathrm{k} \Omega$, valid at low porosity and very close to values measured by Nirmalraj et al. using conductive AFM (1-10 k 2 ) [69]. The $\sim$ tenfold reduction in in-plane conductivity associated with increasing porosity (Fig. 4B) implies that, depending on network morphology, this junction resistance can rise to at least $100 \mathrm{k} \Omega$ for high-resistance junctions. Interestingly, this is close to the mean value of $98 \mathrm{k} \Omega$, reported for junctions between individual singlewalled nanotubes [70], which would be expected to be of low area and so high resistance.

\subsection{Conductivity anisotropy}

We would expect the compression-induced alignment to lead to significant conductivity anisotropy in these films. To explore this, we measured the out-of-plane conductivity (i.e. in the direction of compression, $\sigma_{\mathrm{OOP}}$ ) for a subset of films, finding considerable scatter with values between 8 and $95 \mathrm{~S} / \mathrm{m}$, much lower than the inplane measurements quoted above. We can quantify the conductivity anisotropy via the ratio $\sigma_{\mathrm{IP}} / \sigma_{\mathrm{OOP}}$, which is plotted versus porosity in Fig. 4C. We find a significant change in conductivity anisotropy with porosity, increasing from $\sim 25$ at P 0.7 to $>1000$ at very low porosity, with the latter value in line with that expected for HOPG [71]. This increase in anisotropy as porosity is decreased is, of course, a direct result of the platelet alignment observed in Fig. 3E.

An upper bound for the conductivity anisotropy $\sigma_{\mathrm{IP}} / \sigma_{\mathrm{OOP}}$ can be estimated in a simple and elegant way. As shown in Figs. 1D, 4C and 5 , low porosity leads to nanosheets that tend to be stacked on top of one another. Taking this to the extreme limit of vanishing porosity, the system would correspond to perfectly stacked platelets that are juxtaposed side by side such that there is no void space left in the network (Fig. 3D). In this limit, in-plane and out-of-plane resistances can be easily obtained by simply counting the number of parallel paths, each one of them made of a number of resistors arranged in series. If the film is of dimensions $\mathrm{L} \times \mathrm{L} \times \mathrm{H}$ and the individual nanosheets are $\mathrm{l} \times \mathrm{l} \times \mathrm{h}$ in size, the number of resistors in a conducting path is $\mathrm{L} / \mathrm{l}$ for in-plane conduction and $\mathrm{H} / \mathrm{h}$ for out-ofplane conduction. Similarly, the number of in-plane parallel paths is $\mathrm{HL} / \mathrm{hl}$ while the number of out-of-plane parallel paths is $\mathrm{L}^{2} / \mathrm{l}^{2}$. If the in-plane and out-of-plane junction resistances are the same $\left(\mathrm{R}_{\mathrm{J}}\right)$, then it is simple to show that the conductivities are $\sigma_{I P}=1 / R_{\mathrm{J}} h$ and $\sigma_{O O P}=h / R_{J} l^{2}$ yielding a conductivity anisotropy of:

$\frac{\sigma_{I P}}{\sigma_{O O P}}=\left(\frac{l}{h}\right)^{2}$

The low-porosity conductivity anisotropy was $\sim 2000$, implying a nanosheet aspect ratio of $\sim 50$ which is reasonably close to the expected aspect ratio of $\sim 260 /(0.35 \times 7) \sim 100$. Taking the lowporosity, in-plane conductivity to be $\sim 3 \times 10^{4} \mathrm{~S} / \mathrm{m}$ and using $R_{\mathrm{J}}=1$ / $\sigma_{I P} h$ gives $\mathrm{R}_{\mathrm{J}} \sim 13 \mathrm{k} \Omega$, in reasonable agreement with the estimate above. We can compare this result with the low-porosity films of high-aspect ratio graphene reported by Teng et al. [53] These films had a conductivity of $2.2 \times 10^{5} \mathrm{~S} / \mathrm{m}$ for a mean nanosheet thickness of 4.4 layers yielding $R_{\mathrm{J}} \sim 3 \mathrm{k} \Omega$. This demonstrates that further reductions in contact resistance might be obtained, for example via annealing.

We can compare this anisotropy data with that of Celzard et al. who performed in plane and out of plane conductivity measurements on expanded graphite-based monoliths, although at considerably higher porosities than those achieved in this work [62]. Plotting Celzard's anisotropy data on Fig. 4C shows it follows the same trend as that for our graphene networks. While very few reports exist on conductivity anisotropy in platelet networks, we did find a paper by Balandin et al. on the anisotropy of thermal conductivity in networks of reduced graphene oxide [72]. This ratio of in plane and out of plane thermal conductivity is plotted as the open triangle and sits reasonably near the main trend line. It is possible that the overall trend observed in Fig. $4 \mathrm{C}$ represents a general trend for networks of conducting platelets. If this were the case, it would be possible to use the data in Fig. $4 \mathrm{C}$ to approximately estimate conductivity for a given porosity or conversely to estimate porosity from the ratio of in plane to out of plane conductivity. To facilitate this, we have fitted an empirical curve to the data in Fig. 4B finding $\sigma_{\mathrm{IP}} / \sigma_{\mathrm{OOP}}=10^{\left(3.1-3.2 P^{2.5}\right)}$. 


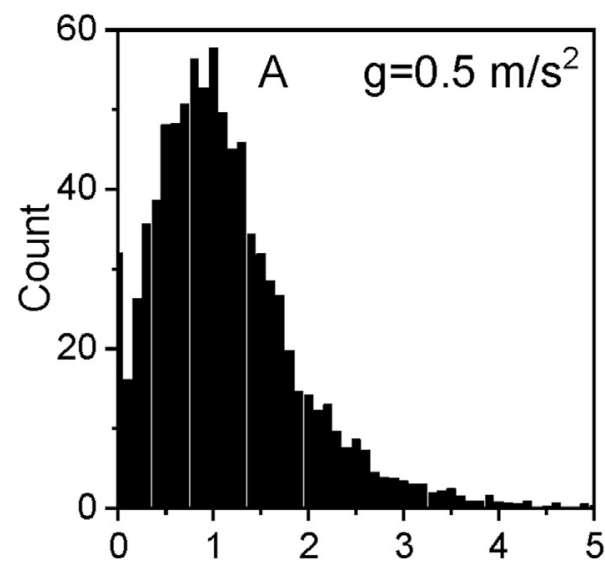

Platelet orientation, $\theta\left(^{\circ}\right)$

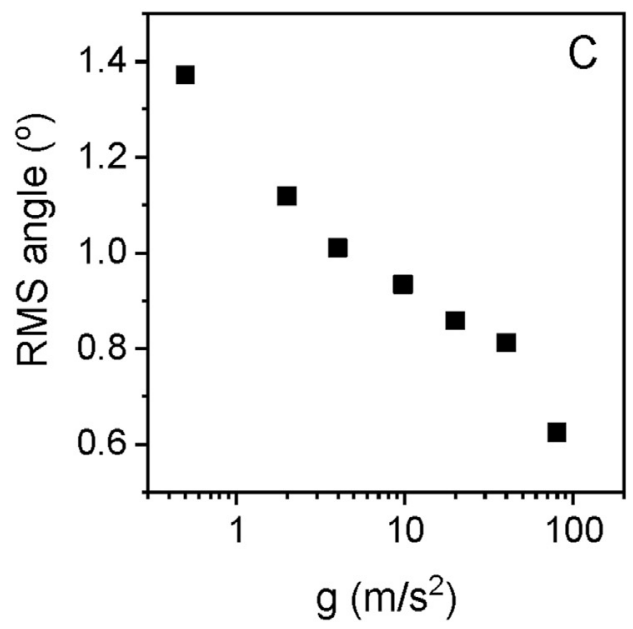

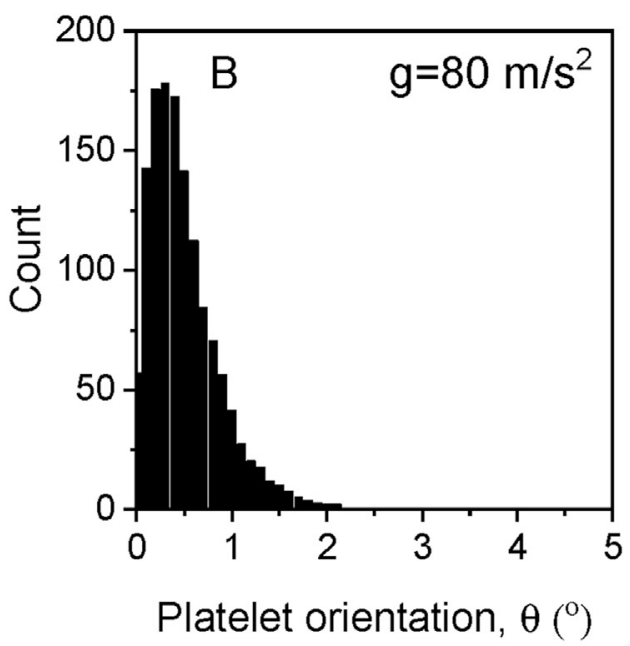

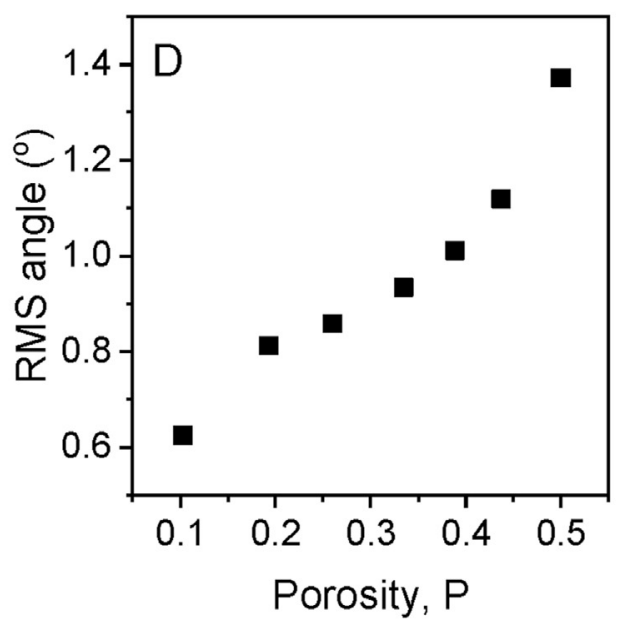

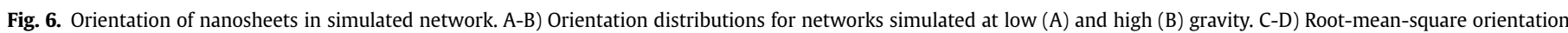
angle plotted versus $C$ ) gravity used in the simulation and D) porosity of the resultant network.

\subsection{Simulating nanoplatelet networks}

Simulations have been carried out to test how the conductivity of a network of conducting platelets changes with the network porosity. One way to account for the effect of the porosity-reducing compression is to implement a deposition simulation in which gravity, $\mathrm{g}$, is a controllable variable. A number of rigid platelets $(\sim 8000)$ of constant size $\left(100 \times 100 \times 0.1\right.$ units $\left.^{3}\right)$ were released from an arbitrary initial height and were allowed to deposit on top of a 2-dimensional square substrate of size $1000 \times 1000$ units $^{2}$ (Fig. 5A). After hitting the substrate, the platelets come to rest. Over time, the system relaxes with the platelets eventually reaching their equilibrium positions, a process which we track by calculating the instantaneous porosity at each time step (Fig. 5B). We note that this simulated network formation, driven by gravity alone can only ever be a crude approximation of network formation. In reality, solution processing means that fluid mechanics-effects will have a very significant impact on network formation. In addition, at the nanoscale van der Waals interactions (which the simulation neglects) cannot be ignored and will certainly have an important influence on network formation.

Within the resultant pile of platelets, we define an internal box (shown as a green rectangle in Fig. 5A) of dimensions $100 \times 100 \times 1.6$ units $^{3}$ for further analysis. From the equilibrium positions of all the platelets within the box, we can calculate a number of parameters. Simplest is the porosity but, as we will see below, we can also obtain information about the platelet orientation, their connectivity and the conductance of the network as a whole. The simulation was performed for 7 different gravity values and was repeated 8 times for each gravity value to reduce fluctuations and improve the statistical significance of our results.

Two cross-section images of internal boxes are shown in Fig. 5C, one for a low- and another for a high-gravity values. These clearly show the networks formed under high gravity to be more aligned and less porous compared to low gravity. As expected, calculated values of network porosity do decrease with gravity as seen in Fig. 5D. As this porosity decrease is clearly linked to the increased alignment at high g, it is worth looking more closely at the platelet orientation.

We calculated the orientation of each platelet via the angle between its normal and the vertical direction. Shown in Fig. 6A and $\mathrm{B}$ are platelet orientation distributions for networks formed at low $\left(\mathrm{g}=0.5 \mathrm{~m} / \mathrm{s}^{2}\right)$ and high $\left(\mathrm{g}=80 \mathrm{~m} / \mathrm{s}^{2}\right)$ gravity. The high gravity distribution is clearly narrower and shifted to lower angles. The root-mean-square (RMS) angle decays quickly with gravity (Fig. 6C), consistent with large numbers of horizontal platelets in the high-g samples. Alternatively, we find the RMS platelet angle to increase significantly with porosity as shown in Fig. 6D. Although 

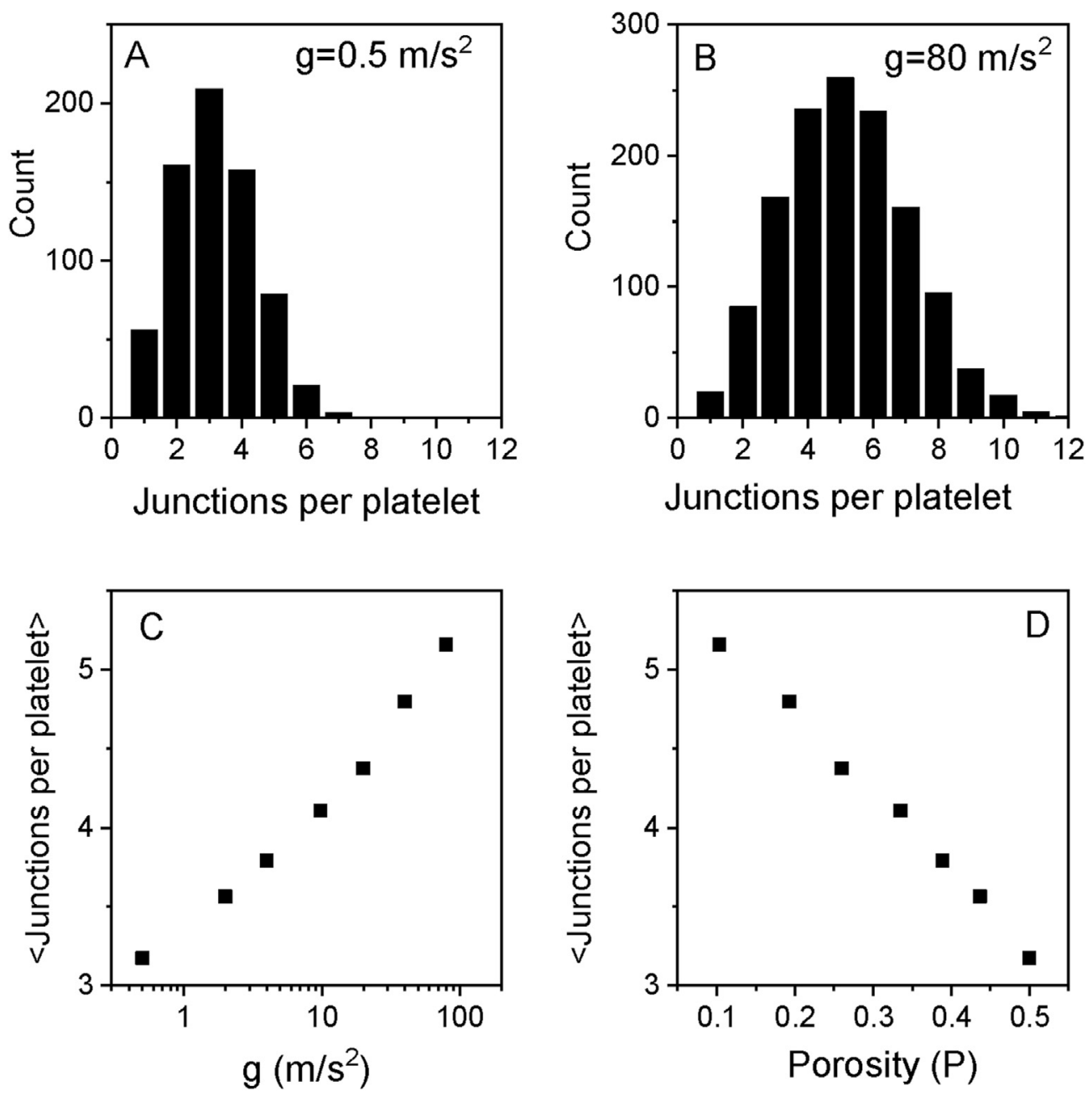

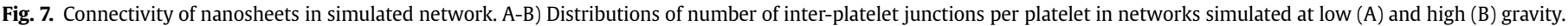
C-D) Mean number of junctions per platelet plotted versus C) gravity used in the simulation and D) porosity of the resultant network.

this data is in qualitative agreement with the experimental data in Fig. 2E, there are some differences. Firstly, the distributions in Fig. 2E are Gaussian distributions centered around zero angle while the distributions in Fig. 6D are lognormal-type distributions with maxima at non-zero angles. This is simply a consequence of how they are measured, with the experimental data measuring the apparent angle between the platelet and the horizontal and the calculated data taking the angle between the normal and the vertical. In addition, we note that while both experimental and simulated angles increase with $\mathrm{P}$, the experimental ones are considerably larger. This disagreement may also partially arise because the simulation assumes rigid platelets whereas the experimental images in Fig. $1 \mathrm{C}$ and D clearly show bending in the platelets. In addition, it may indicate that the real system contains much more disorder than is captured in the simulation, partly because the simulation treats inter-platelet interactions rather crudely.

The simulation can also give information about the connectivity of the network, information that is very difficult to obtain experimentally. To achieve this, we count the number of adjacent platelets each platelet is in contact with. We define this value as the number of junctions per platelet. Shown in Fig. 7A and B are distributions of number of junctions per platelet for networks formed at low $\left(\mathrm{g}=0.5 \mathrm{~m} / \mathrm{s}^{2}\right)$ and high $\left(\mathrm{g}=80 \mathrm{~m} / \mathrm{s}^{2}\right)$ gravity. This clearly shows the network formed at higher $g$ to have more junctions per platelet, indicating higher connectivity. The mean number of junctions per platelet is plotted versus $\mathrm{g}$ in Fig. $7 \mathrm{C}$ and network porosity in Fig. 7D. This data shows a clear falloff from $>5$ junctions per platelet at $\mathrm{P} \sim 0.1$ to $\sim 3$ junctions per platelet at $\mathrm{P} \sim 0.5$. Clearly, the number of junctions per platelet cannot fall much farther without destabilizing the network mechanically. Indeed it has been proposed that ellipsoid particles in jammed systems require at least 4 contacts per particle to be mechanically stable [73]. It is important to emphasize that while the measurements are carried out in specific samples, the simulation consists of averaging over several deposition configurations in order to achieve statistical significance. In other words, while the comparison between simulated and experimental samples is never over networks that have exactly the same connectivity matrix, they are statistically equivalent.

In addition to the number of contacts, we expect their nature to be important. For example, recent work on networks of thick rigid $\mathrm{MoS}_{2}$ nanosheets which interact poorly at junctions, resulting in low-area contacts showed carrier mobilities of $\sim 0.01 \mathrm{~cm}^{2} / \mathrm{Vs}$ [16]. However, networks of very thin $\mathrm{MoS}_{2}$ nanosheets, which were flexible enough to conform to each other to give large area, planar junctions displayed mobilities of $>5 \mathrm{~cm}^{2} / \mathrm{Vs}$ [38]. Thus, the nature of the junctions will have a significant impact on inter-nanosheet charge transfer and so carrier mobility and so conductivity. Here, we use contact area, $A_{J}$, as a proxy for junction resistance, making the assumption that $R_{J} \propto 1 / A_{J}$. 

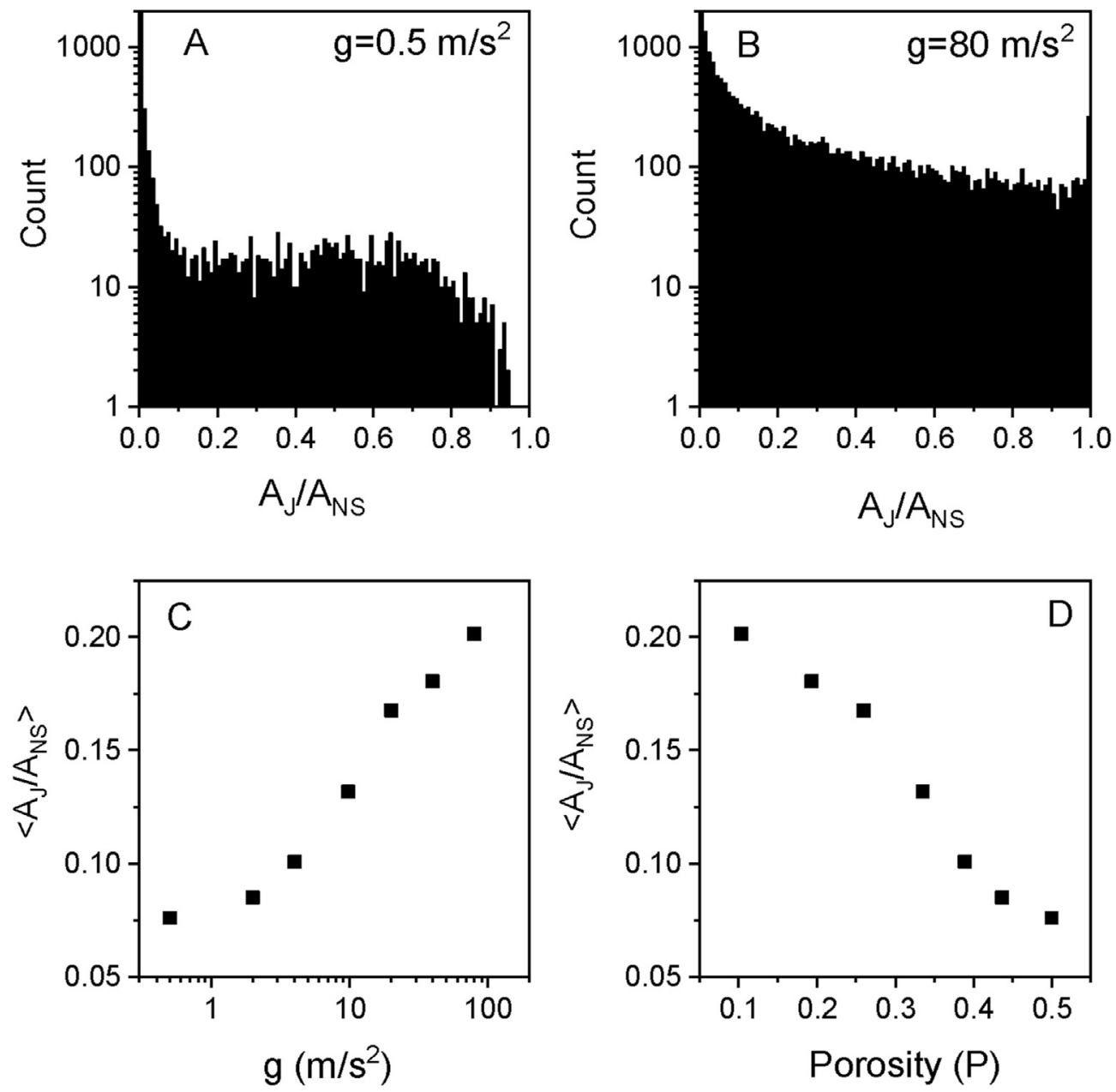

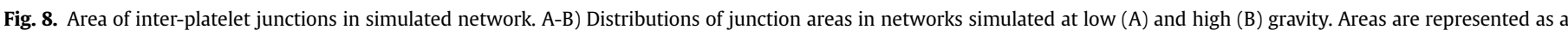
fraction of the platelet area, $A_{J} / A_{T}$. C-D) Mean junctions area plotted versus $C$ ) gravity used in the simulation and $D$ ) porosity of the resultant network.

Fig. 8A and B show the simulated contact area distributions for low- and high-gravity values, respectively (see methods for calculation details). In all cases the contact areas are normalized to the basal plane area of the platelet (i.e. $A_{N S}=100 \times 100$ unit $^{2}$ ). In both cases there is a sharp peak in contacts with very small areas. These represent small-area vertex-plane, vertex-edge, edge-edge or edgeplane contacts. In addition, in both cases there is a tail of larger contact areas $\left(A_{J} / A_{N S}>0.1\right)$. We associate these larger area contacts with plane-plane junctions, where two closely spaced platelets lie parallel over a reasonable area (see Fig. 2C). Such large area contacts $\left(\mathrm{A}_{\mathrm{J}} / \mathrm{A}_{\mathrm{NS}}>0.1\right)$ contribute $\sim 25 \%$ of the total contact area for the low $g$ sample but $\sim 52 \%$ for the high gravity sample. The mean fractional contact area per platelet is plotted versus $\mathrm{g}$ in Fig. $8 \mathrm{C}$ and network porosity in Fig. 8D. We see a considerable increase in $A_{J} / A_{N S}$ with increasing g corresponding to a roughly three-fold decrease going from $\mathrm{P}=0.1$ to $\mathrm{P}=0.5$.

In essence, the data above shows that increasing the effective gravity during network formation effects both the orientation distribution and the network porosity. Those parameters in turn impact the network connectivity and mean junction area. However, most important for practical applications, these final two properties would be expected to impact the network conductivity.

Here we calculate the conductance of the deposited film from the simulations. From the resting positions of the platelets we have full information about the number of contacts that each platelet has with its neighbors, as shown in Fig. 7. Moreover, we can also obtain the junction resistance between touching platelets via $R_{J} \propto 1 / A_{J}$. For example, for two parallel platelets lying on top of one another the junction resistance is much smaller than if they are in contact through their edges or through their vertices. Once the contact area of all junctions are taken into account, we can obtain the conductance of a network made of platelets of intrinsic resistance $R_{P}$ connected to multiple neighbors with respective junction resistances $R_{J}=C / A_{J}$, where $C$ is a constant. Again, we assume that $R_{J}>>R_{\text {nanosheet }}$, which means that the transport across the structure is completely dominated by the network of junction resistances formed between contacting platelets. For the sake of comparison, the parameter $C$ is adjusted so that there is a good agreement between the simulated and experimental in-plane conductivities in the low-porosity limit. Once this is established no further adjustments are needed. For more information, see Methods.

Fig. 9A displays how the calculated in-plane conductivity of the network changes with porosity. We find good qualitative agreement with the overall trend of the conductivity with porosity being well captured by the simulations. However, the simulated conductivity drop with increasing porosity is much larger than that observed in experiments (Fig. 4B). We will return to this below. In addition, out-of-plane conductivity values were also calculated as shown in Fig. 9B and show a significant fall off with porosity. The 

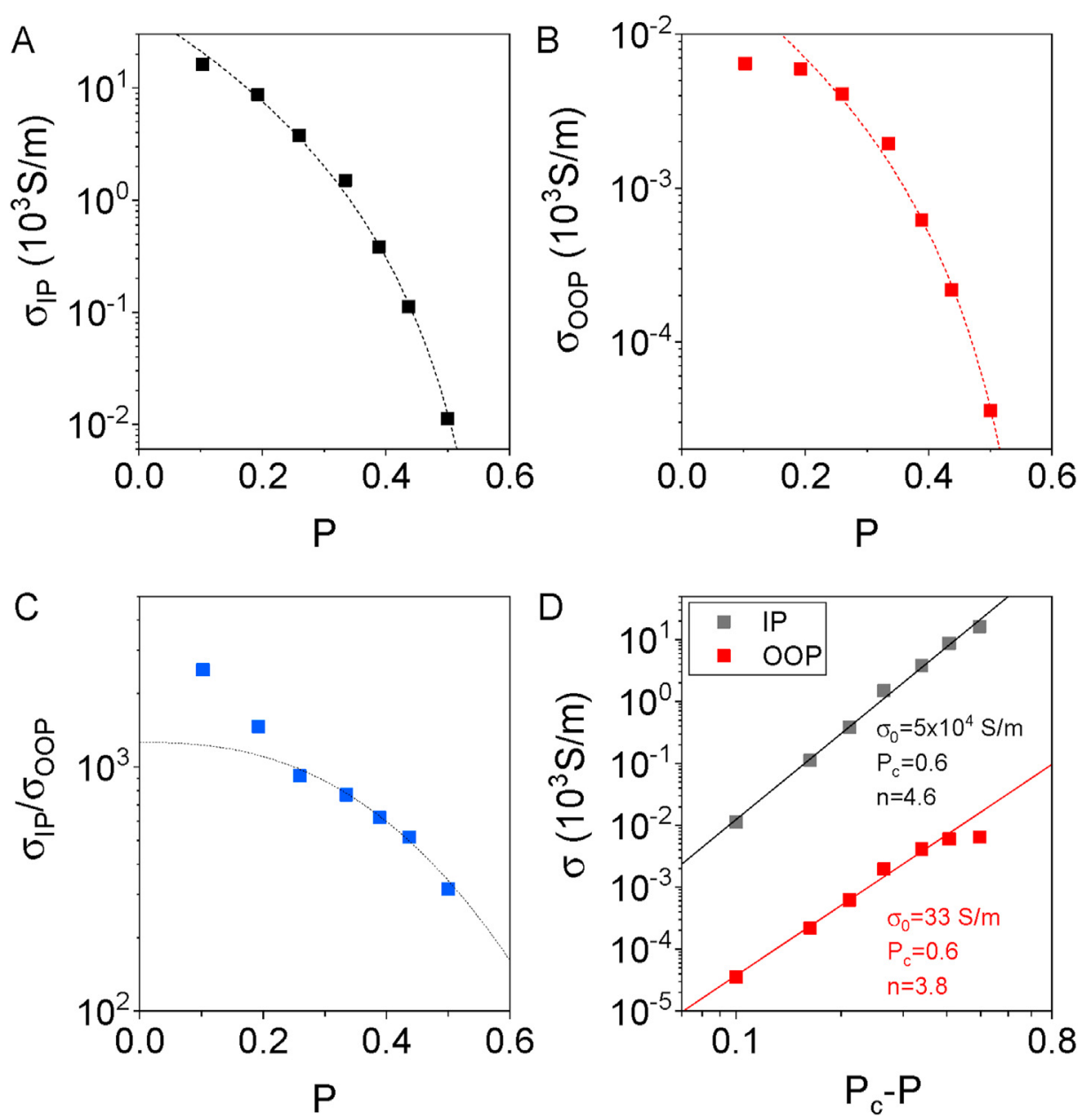

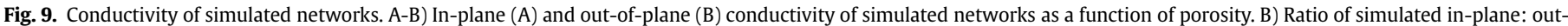

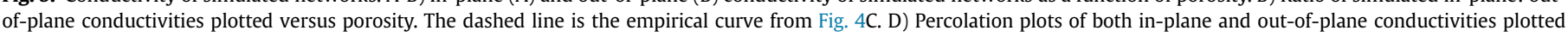
versus $\mathrm{P}_{\mathrm{c}}-\mathrm{P}$. The lines are fits to Eq. (1b) with fit parameters given in the panel.

calculated porosity anisotropy, $\sigma_{\mathrm{IP}} / \sigma_{\mathrm{OOP}}$, is plotted in Fig. $9 \mathrm{C}$ and shows values of $\sim 10^{3}$ as observed experimentally. In fact, we have reproduced the empirical trend-line plotted in Fig. 4C in Fig. 9C. Surprisingly, we find very good agreement with the simulation data.

In principle, the simulation data should be described by percolation theory in a manner similar to the experimental data. Shown in Fig. 9D are percolation plots of both simulated in-plane and outof-plane conductivities versus $\mathrm{P}_{\mathrm{c}}-\mathrm{P}$. In each case, we find reasonably good agreement for $P_{c}=0.6$. The main difference between these fits and the experimental fit in Fig. $4 \mathrm{~B}$ is the fact that the percolation exponents associated with the fits in Fig. 9D have considerably higher exponents compared to the experimental fit. As mentioned above, higher percolation exponents generally indicate broader distributions of junction resistances [67,68]. This may indicate that the junction area distributions in Fig. 8 A-B are broader than would be the case in real systems. We ascribe this to the assumption of non-flexible platelets in the simulations.

\section{Conclusions}

In order to fully characterize and understand the effects of changing morphology on nanosheet networks, a method for controlling and characterizing network porosity has been investigated and outlined allowing access to a porosity range from $~ 70 \%$ to $\sim 14 \%$. It has been shown that compression of these networks results in two key changes: as porosity decreases, the platelets within the network begin to align perpendicularly to the direction of compression and the large pores $(>40 \mathrm{~nm})$ are eliminated while the small pores $(<40 \mathrm{~nm}$ ) remain. Computational studies show that, on the nanoscale, compressed networks tend to have higher degrees of connectivity and lower resistance junctions. These changes result in a significant increase in conductivity as the networks are compressed and the porosity decreases.

From this study it has been clearly demonstrated that the conductivity of a nanosheet sheet network of graphene can be improved by an order of magnitude simply via compression. This improvement in conductivity can be attributed to the removal of large pores from within the network, reducing the inter-flake junction distance. In addition to this it can be seen the film surface areas remain unaffected by the compression treatment, allowing this treatment to be applicable to catalysis applications. Finally, the compression treatment has been shown to further align the platelets as seen with a combination of SEM orientation distributions in addition to IP/OOP conductivity changes. This alignment within the nanosheet network as well as the removal of large pores is responsible for the increase in the IP conductivity of the network. 


\section{CRediT authorship contribution statement}

Sebastian Barwich: Investigation, Writing - review \& editing. João Medeiros de Araújo: Investigation, Methodology, Software. Aran Rafferty: Investigation, Resources. Claudia Gomes da Rocha: Investigation, Methodology, Software. Mauro S. Ferreira: Methodology, Formal analysis, Writing - original draft, Supervision. Jonathan N. Coleman: Conceptualization, Formal analysis, Writing - original draft, Writing - review \& editing, Supervision.

\section{Declaration of competing interest}

The authors declare that they have no known competing financial interests or personal relationships that could have appeared to influence the work reported in this paper.

\section{Acknowledgements}

We acknowledge Science Foundation Ireland (SFI, SFI/12/RC/ 2278), the European Commission ( $n^{\circ} 696656$, Graphene Flagship), the European Research Council (FUTURE-PRINT) and Nokia Bell labs (Ireland) for funding. We also acknowledge The Research IT Unit at Trinity College Dublin, the WestGrid (www.westgrid.ca) and Compute Canada Calcul Canada (www.computecanada.ca) for computational resources. J.M.A. acknowledges CNPq for support through Bolsa de Produtividade em Pesquisa No.313431/2018-3.

\section{References}

[1] F. Bonaccorso, A. Bartolotta, J.N. Coleman, C. Backes, 2D-Crystal-Based functional inks, Adv. Mater. 28 (29) (2016) 6136-6166.

[2] L.Y. Niu, J.N. Coleman, H. Zhang, H. Shin, M. Chhowalla, Z.J. Zheng, Production of two-dimensional nanomaterials via liquid-based direct exfoliation, Small 12 (3) (2016) 272-293.

[3] V. Nicolosi, M. Chhowalla, M.G. Kanatzidis, M.S. Strano, J.N. Coleman, Liquid exfoliation of layered materials, Science 340 (2013) 6139.

[4] M. Lotya, Y. Hernandez, P.J. King, R.J. Smith, V. Nicolosi, L.S. Karlsson, F.M. Blighe, S. De, Z. Wang, I.T. McGovern, G.S. Duesberg, J.N. Coleman, Liquid phase production of graphene by exfoliation of graphite in surfactant/water solutions, J. Am. Chem. Soc. 131 (10) (2009) 3611-3620.

[5] J.W.T. Seo, A.A. Green, A.L. Antaris, M.C. Hersam, High-concentration aqueous dispersions of graphene using nonionic, biocompatible block copolymers, J. Phys. Chem. Lett. 2 (9) (2011) 1004-1008.

[6] S.M. Notley, Highly concentrated aqueous suspensions of graphene through ultrasonic exfoliation with continuous surfactant addition, Langmuir 28 (40) (2012) 14110-14113.

[7] Y. Hernandez, M. Lotya, D. Rickard, S.D. Bergin, J.N. Coleman, Measurement of multicomponent solubility parameters for graphene facilitates solvent discovery, Langmuir 26 (5) (2010) 3208-3213.

[8] K.R. Paton, E. Varrla, C. Backes, R.J. Smith, U. Khan, A. O’Neill, C. Boland, M. Lotya, O.M. Istrate, P. King, T. Higgins, S. Barwich, P. May, P. Puczkarski, I. Ahmed, M. Moebius, H. Pettersson, E. Long, J. Coelho, S.E. O'Brien, E.K. McGuire, B.M. Sanchez, G.S. Duesberg, N. McEvoy, T.J. Pennycook, C. Downing, A. Crossley, V. Nicolosi, J.N. Coleman, Scalable production of large quantities of defect-free few-layer graphene by shear exfoliation in liquids, Nat. Mater. 13 (6) (2014) 624-630.

[9] G. Cunningham, M. Lotya, C.S. Cucinotta, S. Sanvito, S.D. Bergin, R. Menzel, M.S.P. Shaffer, J.N. Coleman, Solvent exfoliation of transition metal dichalcogenides: dispersibility of exfoliated nanosheets varies only weakly between compounds, ACS Nano 6 (4) (2012) 3468-3480.

[10] A.B. Bourlinos, V. Georgakilas, R. Zboril, T.A. Steriotis, A.K. Stubos, Liquidphase exfoliation of graphite towards solubilized graphenes, Small 5 (2009) 1841.

[11] J.N. Coleman, M. Lotya, A. O’Neill, S.D. Bergin, P.J. King, U. Khan, K. Young, A. Gaucher, S. De, R.J. Smith, I.V. Shvets, S.K. Arora, G. Stanton, H.Y. Kim, K. Lee, G.T. Kim, G.S. Duesberg, T. Hallam, J.J. Boland, J.J. Wang, J.F. Donegan, J.C. Grunlan, G. Moriarty, A. Shmeliov, R.J. Nicholls, J.M. Perkins, E.M. Grieveson, K. Theuwissen, D.W. McComb, P.D. Nellist, V. Nicolosi, Twodimensional nanosheets produced by liquid exfoliation of layered materials, Science 331 (6017) (2011) 568-571.

[12] D. Hanlon, C. Backes, E. Doherty, C.S. Cucinotta, N.C. Berner, C. Boland, K. Lee, A. Harvey, P. Lynch, Z. Gholamvand, S.F. Zhang, K.P. Wang, G. Moynihan, A. Pokle, Q.M. Ramasse, N. McEvoy, W.J. Blau, J. Wang, G. Abellan, F. Hauke, A. Hirsch, S. Sanvito, D.D. O’Regan, G.S. Duesberg, V. Nicolosi, J.N. Coleman, Liquid exfoliation of solvent-stabilized few-layer black phosphorus for applications beyond electronics, Nat. Commun. 6 (2015), 8563.
[13] A. Harvey, C. Backes, Z. Gholamvand, D. Hanlon, D. McAteer, H.C. Nerl, E. McGuire, A. Seral-Ascaso, O.M. Ramasse, N. McEvoy, S. Winters, N.C. Berner, D. McCloskey, J.F. Donegan, G.S. Duesberg, V. Nicolosi, J.N. Coleman, Preparation of gallium sulfide nanosheets by liquid exfoliation and their application as hydrogen evolution catalysts, Chem. Mater. 27 (9) (2015) 3483-3493.

[14] C. Backes, D. Campi, B.M. Szydlowska, K. Synnatschke, E. Ojala, F. Rashvand, A. Harvey, A. Griffin, Z. Sofer, N. Marzari, J.N. Coleman, D.D. O’Regan, Equipartition of energy defines the size-thickness relationship in liquid-exfoliated nanosheets, ACS Nano 13 (6) (2019) 7050-7061.

[15] C. Gabbett, C.S. Boland, A. Harvey, V. Vega-Mayoral, R.J. Young, J.N. Coleman, The effect of network formation on the mechanical properties of 1D:2D nano: nano composites, Chem. Mater. 30 (15) (2018) 5245-5255.

[16] A.G. Kelly, T. Hallam, C. Backes, A. Harvey, A.S. Esmaeily, I. Godwin, J. Coelho, V. Nicolosi, J. Lauth, A. Kulkarni, S. Kinge, L.D.A. Siebbeles, G.S. Duesberg, J.N. Coleman, All-printed thin-film transistors from networks of liquidexfoliated nanosheets, Science 356 (6333) (2017) 69-73.

[17] C.S. Boland, U. Khan, G. Ryan, S. Barwich, R. Charifou, A. Harvey, C. Backes, Z. Li, M.S. Ferreira, M.E. Mobius, R.J. Young, J.N. Coleman, Sensitive electromechanical sensors using viscoelastic graphene-polymer nanocomposites, Science 354 (6317) (2016) 1257-1260.

[18] T. Carey, S. Cacovich, G. Divitini, J.S. Ren, A. Mansouri, J.M. Kim, C.X. Wang, C. Ducati, R. Sordan, F. Torrisi, Fully inkjet-printed two-dimensional material field-effect heterojunctions for wearable and textile electronics, Nat. Commun. 8 (2017), 1202.

[19] C. Casiraghi, M. Macucci, K. Parvez, R. Worsley, Y. Shin, F. Bronte, C. Borri, M. Paggi, G. Fiori, Inkjet printed 2D-crystal based strain gauges on paper, Carbon 129 (2018) 462-467.

[20] X.W. Feng, Y.D. Li, L. Wang, S. Chen, Z.G. Yu, E.E.C. Tan, N. Macadam, G.H. Hu, L. Huang, L. Chen, X. Gong, D.Z. Chi, T. Hasan, A.V.Y. Thean, Y.W. Zhang, K.W. Ang, A fully printed flexible MoS2 memristive artificial synapse with femtojoule switching energy, Advanced Electronic Materials 5(12) 1900740.

[21] D. McManus, S. Vranic, F. Withers, V. Sanchez-Romaguera, M. Macucci, H.F. Yang, R. Sorrentino, K. Parvez, S.K. Son, G. Iannaccone, K. Kostarelos, G. Fiori, C. Casiraghi, Water-based and biocompatible 2D crystal inks for allinkjet-printed heterostructures, Nat. Nanotechnol. 12 (4) (2017) 343-350.

[22] S.Y. Qiang, T. Carey, A. Arbab, W.H. Song, C.X. Wang, F. Torrisi, Wearable solidstate capacitors based on two-dimensional material all-textile heterostructures, Nanoscale 11 (20) (2019) 9912-9919.

[23] R. Worsley, L. Pimpolari, D. McManus, N. Ge, R. Ionescu, J.A. Wittkopf, A. Alieva, G. Basso, M. Macucci, G. Iannaccone, K.S. Novoselov, H. Holder, G. Fiori, C. Casiraghi, All-2D material inkjet-printed capacitors: toward fully printed integrated circuits, ACS Nano 13 (1) (2019) 54-60.

[24] T.C. Wu, A. De Luca, Q.Y. Zhong, X.X. Zhu, O. Ogbeide, D.S. Um, G.H. Hu, T. Albrow-Owen, F. Udrea, T. Hasan, Inkjet-printed CMOS-integrated graphene metal oxide sensors for breath analysis, Npj 2d Materials and Applications 3 (2019) 42.

[25] S. Witomska, T. Leydecker, A. Ciesielski, P. Samori, Production and patterning of liquid phase-exfoliated 2D sheets for applications in optoelectronics, Adv. Funct. Mater. 29 (22) (2019) 23.

[26] M.J. Large, S.P. Ogilvie, A. Amorim Graf, P.J. Lynch, M.A. O’Mara, T. Waters, I. Jurewicz, J.P. Salvage, A.B. Dalton, Large-scale surfactant exfoliation of graphene and conductivity-optimized graphite enabling wireless connectivity, Adv. Mater. Technol. 11.

[27] P. He, J.Y. Cao, H. Ding, C.G. Liu, J. Neilson, Z.L. Li, I.A. Kinloch, B. Derby, Screenprinting of a highly conductive graphene ink for flexible printed electronics, ACS Appl. Mater. Interfaces 11 (35) (2019) 32225-32234.

[28] L. Kavan, J.H. Yum, M. Gratzel, Optically transparent cathode for dyesensitized solar cells based on graphene nanoplatelets, ACS Nano 5 (2011) $165-172$.

[29] J.-Z. Wang, L. Lu, M. Lotya, J.N. Coleman, S.-L. Chou, H.-K. Liu, A.I. Minett, J. Chen, Development of MoS2-CNT composite thin film from layered MoS2 for lithium batteries, Advanced Energy Materials 3 (6) (2013) 798-805.

[30] V. Dua, S.P. Surwade, S. Ammu, S.R. Agnihotra, S. Jain, K.E. Roberts, S. Park, R.S. Ruoff, S.K. Manohar, All-organic vapor sensor using inkjet-printed reduced graphene oxide, Angew. Chem. Int. Ed. 49 (12) (2010) 2154-2157.

[31] D. Kong, L.T. Le, Y. Li, J.L. Zunino, W. Lee, Temperature-dependent electrical properties of graphene inkjet-printed on flexible materials, Langmuir 28 (37) (2012) 13467-13472.

[32] D.J. Finn, M. Lotya, G. Cunningham, R.J. Smith, D. McCloskey, J.F. Donegan, J.N. Coleman, Inkjet deposition of liquid-exfoliated graphene and MoS2 nanosheets for printed device applications, J. Mater. Chem. C 2 (2014) 925-932.

[33] Y. Xu, I. Hennig, D. Freyberg, A.J. Strudwick, M.G. Schwab, T. Weitz, K.C.-P. Cha, Inkjet-printed energy storage device using graphene/polyaniline inks, J. Power Sources 248 (2014) 483-488.

[34] A.G. Kelly, V. Vega-Mayoral, J.B. Boland, J.N. Coleman, Whiskey-phase exfoliation: exfoliation and printing of nanosheets using Irish whiskey, 2D Mater. 6 (4) (2019), 045036.

[35] D. Song, A. Mahajan, E.B. Secor, M.C. Hersam, L.F. Francis, C.D. Frisbie, Highresolution transfer printing of graphene lines for fully printed, flexible electronics, ACS Nano 11 (7) (2017) 7431-7439.

[36] A.C.M. de Moraes, W.J. Hyun, J.W.T. Seo, J.R. Downing, J.M. Lim, M.C. Hersam, Ion-conductive, viscosity-tunable hexagonal boron nitride nanosheet inks, Adv. Funct. Mater. 29 (39) (2019), 1902245.

[37] B. Gupta, H. Matte, Solution-processed layered hexagonal boron nitride 
dielectrics: a route toward fabrication of high performance flexible devices, ACS Appl. Electron. Mater. 1 (10) (2019) 2130-2139.

[38] Z.Y. Lin, Y. Liu, U. Halim, M.N. Ding, Y.Y. Liu, Y.L. Wang, C.C. Jia, P. Chen, X.D. Duan, C. Wang, F. Song, M.F. Li, C.Z. Wan, Y. Huang, X.F. Duan, Solutionprocessable 2D semiconductors for high-performance large-area electronics, Nature 562 (7726) (2018) 254-258.

[39] D. Lam, K.S. Chen, J. Kang, X.L. Liu, M.C. Hersam, Anhydrous liquid-phase exfoliation of pristine electrochemically active GeS nanosheets, Chem. Mater. 30 (7) (2018) 2245-2250.

[40] A. Harvey, X.Y. He, I.J. Godwin, C. Backes, D. McAteer, N.C. Berner, N. McEvoy, A. Ferguson, A. Shmeliov, M.E.G. Lyons, V. Nicolosi, G.S. Duesberg, J.F. Donegan, J.N. Coleman, Production of $\mathrm{Ni}(\mathrm{OH})(2)$ nanosheets by liquid phase exfoliation: from optical properties to electrochemical applications, J. Mater. Chem. 4 (28) (2016) 11046-11059.

[41] E.B. Secor, M.H. Dos Santos, S.G. Wallace, N.P. Bradshaw, M.C. Hersam, Tailoring the porosity and microstructure of printed graphene electrodes via polymer phase inversion, J. Phys. Chem. C 122 (25) (2018) 13745-13750.

[42] M. Alsaif, A.F. Chrimes, T. Daeneke, S. Balendhran, D.O. Bellisario, Y. Son, M.R. Field, W. Zhang, H. Nili, E.P. Nguyen, K. Latham, J. van Embden, M.S. Strano, J.Z. Ou, K. Kalantar-zadeh, High-Performance field effect transistors using electronic inks of 2D molybdenum oxide nanoflakes, Adv. Funct. Mater. 26 (1) (2016) 91-100.

[43] J. Perelaer, P.J. Smith, C.E. Hendriks, A.M.J. van den Berg, U.S. Schubert, The preferential deposition of silica micro-particles at the boundary of inkjet printed droplets, Soft Matter 4 (5) (2008) 1072-1078.

[44] X. Shen, C.-M. Ho, T.-S. Wong, Minimal size of coffee ring structure, J. Phys. Chem. B 114 (16) (2010) 5269-5274.

[45] S. Vittorio, C. Richard, L.P. E, R. David, C.J. N, Spray deposition of highly transparent, low-resistance networks of silver nanowires over large areas, Small 7 (18) (2011) 2621-2628.

[46] P. Jolke, P.J. S, M.M.P. W, v.d.B. Erwin, E. Rebecca, P.H.J.M K, U.S. S, Droplet tailoring using evaporative inkjet printing, Macromol. Chem. Phys. 210 (5) (2009) 387-393.

[47] S. Madhusudan, H.H. M, D. Parul, J.G. E, Inkjet printing-process and its applications, Adv. Mater. 22 (6) (2010) 673-685.

[48] F. Torrisi, T. Hasan, W. Wu, Z. Sun, A. Lombardo, T.S. Kulmala, G.-W. Hsieh, S. Jung, F. Bonaccorso, P.J. Paul, D. Chu, A.C. Ferrari, Inkjet-printed graphene electronics, ACS Nano 6 (4) (2012) 2992-3006.

[49] B. Muchharla, T.N. Narayanan, K. Balakrishnan, P.M. Ajayan, S. Talapatra, Temperature dependent electrical transport of disordered reduced graphene oxide, 2D Mater. 1 (1) (2014).

[50] T.M. Higgins, S. Finn, M. Matthiesen, S. Grieger, K. Synnatschke, M. Brohmann, M. Rother, C. Backes, J. Zaumseil, Electrolyte-gated n-type transistors produced from aqueous inks of WS2 nanosheets, Adv. Funct. Mater. 29 (4) (2019), 1804387.

[51] D. O'Suilleabhain, V. Vega-Mayoral, A.G. Kelly, A. Harvey, J.N. Coleman, Percolation effects in electrolytically gated WS2/graphene nano:nano composites, ACS Appl. Mater. Interfaces 11 (8) (2019) 8545-8555.

[52] S. Barwich, J.N. Coleman, M.E. Mobius, Yielding and flow of highly concentrated, few-layer graphene suspensions, Soft Matter 11 (16) (2015) 3159-3164.

[53] C. Teng, D. Xie, J.F. Wang, Z. Yang, G.Y. Ren, Y. Zhu, Ultrahigh conductive graphene paper based on ball-milling exfoliated graphene, Adv. Funct. Mater. 27 (20) (2017) 1700240.

[54] J.A. Fairfield, C.G. Rocha, C. O'Callaghan, M.S. Ferreira, J.J. Boland, Co-percolation to tune conductive behaviour in dynamical metallic nanowire networks, Nanoscale 8 (43) (2016) 18516-18523.

[55] H.G. Manning, F. Niosi, C.G. Rocha, A.T. Bellew, C. O’Callaghan, S. Biswas, P.F. Flowers, B.J. Wiley, J.D. Holmes, M.S. Ferreira, J.J. Boland, Emergence of winner-takes-all connectivity paths in random nanowire networks, Nat. Commun. 9 (2018) 3219.

[56] C. Backes, K.R. Paton, D. Hanlon, S. Yuan, M.I. Katsnelson, J. Houston, R.J. Smith, D. McCloskey, J.F. Donegan, J.N. Coleman, Spectroscopic metrics allow in situ measurement of mean size and thickness of liquid-exfoliated few-layer graphene nanosheets, Nanoscale 8 (7) (2016) 4311-4323.

[57] Y. Hernandez, V. Nicolosi, M. Lotya, F.M. Blighe, Z. Sun, S. De, M. T, B. Holland, M. Byrne, Y.K. Gun'Ko, J.J. Boland, P. Niraj, G. Duesberg, S. Krishnamurthy, R. Goodhue, J. Hutchison, V. Scardaci, A.C. Ferrari, J.N. Coleman, High-yield production of graphene by liquid-phase exfoliation of graphite, Nat. Nanotechnol. 3 (9) (2008) 563-568.

[58] J.-Y. Hong, J. Kong, S.H. Kim, Spatially controlled graphitization of reduced graphene oxide films via a green mechanical approach, Small 10 (23) (2014) 4839-4844.

[59] K.S.W. Sing, D.H. Everett, R.A.W. Haul, L. Moscou, R.A. Pierotti, J. Rouquerol, T. Siemieniewska, Reporting physisorption data for gas/solid systems with special reference to the determination of surface area and porosity, Pure Appl. Chem. 57 (4) (1985) 603-619.

[60] J. Rouquerol, G.V. Baron, R. Denoyel, H. Giesche, J. Groen, P. Klobes, P. Levitz, A.V. Neimark, S. Rigby, R. Skudas, K. Sing, M. Thommes, K. Unger, The characterization of macroporous solids: an overview of the methodology, Microporous Mesoporous Mater. 154 (2012) 2-6.

[61] K. Meyer, P. Klobes, Comparison between different presentations of pore size distribution in porous materials, Fresen. J. Anal. Chem. 363 (2) (1999) $174-178$.

[62] A. Celzard, J.F. Marêché, G. Furdin, S. Puricelli, Electrical conductivity of anisotropic expanded graphite-based monoliths, J. Phys. Appl. Phys. 33 (23) (2000) 3094.

[63] G. Cunningham, M. Lotya, N. McEvoy, G.S. Duesberg, P. van der Schoot, J.N. Coleman, Percolation scaling in composites of exfoliated MoS2 filled with nanotubes and graphene, Nanoscale 4 (20) (2012) 6260-6264.

[64] M. Mecklenburg, A. Schuchardt, Y.K. Mishra, S. Kaps, R. Adelung, A. Lotnyk, L. Kienle, K. Schulte, Aerographite: Ultra lightweight, flexible nanowall, carbon microtube material with outstanding mechanical performance, Adv. Mater. 24 (26) (2012) 3486-3490.

[65] D. Stauffer, A. Aharony, Introduction to Percolation Theory, CRC Press, 1994.

[66] W. Bauhofer, J.Z. Kovacs, A review and analysis of electrical percolation in carbon nanotube polymer composites, Compos. Sci. Technol. 69 (10) (2009) 1486-1498.

[67] I. Balberg, Limits on the continuum-percolation transport exponents, Phys. Rev. B 57 (21) (1998) 13351-13354.

[68] N. Johner, C. Grimaldi, I. Balberg, P. Ryser, Transport exponent in a threedimensional continuum tunneling-percolation model, Phys. Rev. B 77 (17) (2008), 174204.

[69] P.N. Nirmalraj, T. Lutz, S. Kumar, G.S. Duesberg, J.J. Boland, Nanoscale mapping of electrical resistivity and connectivity in graphene strips and networks, Nano Lett. 11 (1) (2011) 16-22.

[70] P.N. Nirmalraj, P.E. Lyons, S. De, J.N. Coleman, J.J. Boland, Electrical connectivity in single-walled carbon nanotube networks, Nano Lett. 9 (11) (2009) 3890-3895.

[71] D. Marchand, C. Fretigny, C.M. Lagues, A.P. Legrand, E. McRae, J.F. Mareche, M. Lelaurain, Surface structure and electrical conductivity of natural and artificial graphites, Carbon 22 (1984) 497-506.

[72] J.D. Renteria, S. Ramirez, H. Malekpour, B. Alonso, A. Centeno, A. Zurutuza, A.I. Cocemasov, D.L. Nika, A.A. Balandin, Strongly anisotropic thermal conductivity of free-standing reduced graphene oxide films annealed at high temperature, Adv. Funct. Mater. 25 (29) (2015) 4664-4672.

[73] M. van Hecke, Jamming of soft particles: geometry, mechanics, scaling and isostaticity, J. Phys. Condens. Matter 22 (3) (2010), 033101. 